Document downloaded from:

http://hdl.handle.net/10251/90954

This paper must be cited as:

Reynoso Meza, G.; Blasco Ferragud, FX.; Sanchís Saez, J.; Herrero Durá, JM. (2013). Comparison of design concepts in multi-criteria decision-making using level diagrams. INFORMATION SCIENCES. 221(1):124-141. doi:10.1016/j.ins.2012.09.049

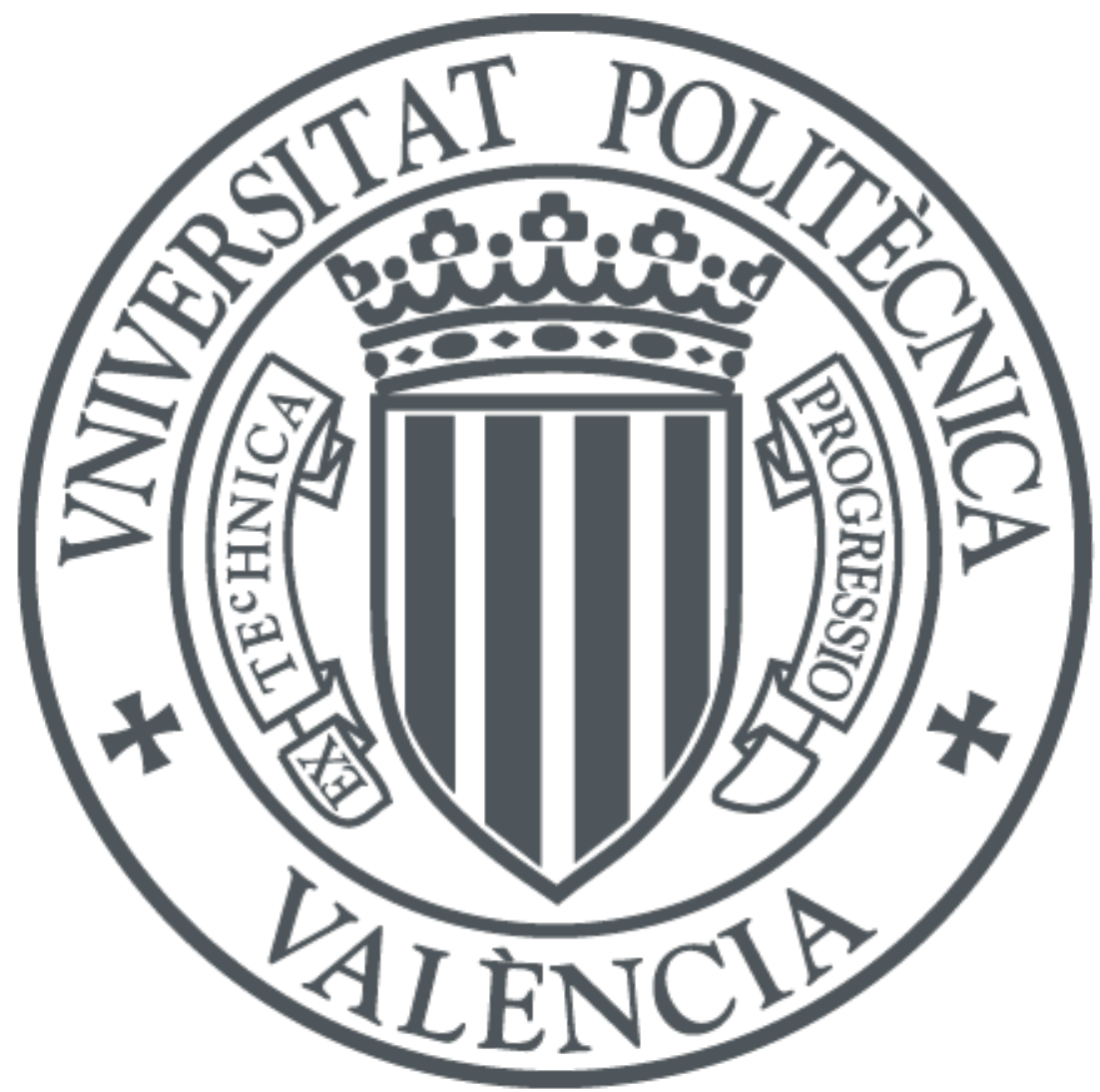

The final publication is available at

https://doi.org/10.1016/j.ins.2012.09.049

Copyright ELSEVIER SCIENCE INC

Additional Information 


\title{
Comparison of Design Concepts in Multi-Criteria Decision-Making Using Level Diagrams
}

\author{
Gilberto Reynoso-Meza $^{\mathrm{a}, *}$, Xavier Blasco ${ }^{\mathrm{a}}$, Javier Sanchis ${ }^{\mathrm{a}}$, Juan M. Herrero ${ }^{\mathrm{a}}$ \\ ${ }^{a}$ Instituto Universitario de Automática e Informática Industrial, Universitat Politècnica de València, \\ Camino de Vera $s / n$, Valencia 46022, Spain
}

\begin{abstract}
In this work, we address the evaluation of design concepts and the analysis of multiple Pareto fronts in multi-criteria decision-making using level diagrams. Such analysis is relevant when two (or more) design concepts with different design alternatives lie in the same objective space, but describe different Pareto fronts. Therefore, the problem can be stated as a Pareto front comparison between two (or more) design concepts that only differ in their relative complexity, implementation issues, or the theory applied to solve the problem at hand. Such analysis will help the decision maker obtain a better insight of a conceptual solution and be able to decide if the use of a complex concept is justified instead of a simple concept. The approach is validated in a set of multi-criteria decision making benchmark problems.
\end{abstract}

Keywords: multi-criteria decision-making, multi-objective optimization, decision-making tools, m-dimensional Pareto front comparison, m-dimensional Pareto front visualization, s-Pareto front.

\section{Introduction}

It is common to state a design problem as an optimization statement, where a specific cost index must be optimized. However, many real world problems require the fulfillment of a set of requirements and specifications. In that case, it is said to have a multi-objective problem (MOP) instead of a single-objective problem. In such statements, it is usual to find that some objectives are in conflict with each other, and a trade-off solution must be found (or selected).

Multi-objective optimization (MOO) can handle these issues in a simple manner, due to its simultaneous optimization approach. In MOO, all the objectives are significant to the designer, and as a consequence, each is optimized. In general, there is no single solution because no solution is better than the others in all the objectives. Therefore, a set of solutions, the Pareto set $\boldsymbol{\Theta}_{\boldsymbol{P}}$, is defined and its objective vector set as the Pareto front $\boldsymbol{J}_{\boldsymbol{P}}$. This set of solutions offers the decision maker (DM) greater flexibility at the multi-criteria

\footnotetext{
*Corresponding author. Tel.:+34963877007;fax:+34963879579

Email address: gilreyme@posgrado.upv.es (Gilberto Reynoso-Meza)

$U R L$ : http://cpoh.upv.es/ (Gilberto Reynoso-Meza)
} 
decision-making stage. The role of the designer is to select the best solution according to her/his needs and preferences for a particular situation.

MOO techniques search for a discrete approximation $\Theta_{P}^{*}$ of the Pareto set $\Theta_{P}$ in order to build a useful description $\boldsymbol{J}_{\boldsymbol{P}}^{*}$ of the Pareto front that is as good as possible. In this way, the DM has a set of solutions for a given problem and a high degree of flexibility when choosing a particular or desired solution. Classic techniques [31] for building this Pareto front have been used (for example: weighting vectors; $\epsilon$-constraint and goal programming methods); as well as specialized algorithms (normal boundary intersection method [8], normal constraint method [29, 42, 27, 26]; and successive Pareto front optimization [40]). Multi-objective evolutionary algorithms (MOEAs) have been recently used due to their flexibility when dealing with non-convex and highly constrained functions [7, 6]. Some examples include: NSGA-II [10]; MOGA [13]; ev-MOGA [17]; pa $\epsilon$-MyDE [16]; pa $\epsilon$-ODEMO [14], sp-MODE [35], NOSGA [11], DNMOEA/HI [24]. Furthermore, new techniques to improve diversity in the Pareto front for MOEAs have been proposed [47, 34, 5].

Once the DM has been provided with a Pareto front $\boldsymbol{J}_{P}^{*}$, the DM will need to analyze the trade-off between objectives and select the most preferable solution according to his preferences. Such an approach has been termed generate first-choose later [30]. Several techniques and methods have been developed to facilitate the DM's task [12, 1, 32, 49]. It is widely accepted that visualization tools are valuable and provide decision makers with a meaningful method to analyze the Pareto front and take decisions [3]. We can recall the desirable characteristics for such visualization techniques noted in [25] included: simplicity (it should be easy to understand); persistence (the DM should be able to retain all information in his/her mind); and completeness (all the relevant information should be shown). Moreover, desirable characteristics (at software level) include interactivity with the DM and an intuitive graphical user interface.

For two-dimensional problems (and sometimes for three-dimensional problems) it is usually straightforward to make an accurate graphical analysis of the Pareto front, but the difficulty increases with the dimension of the problem. Common alternatives to tackle an analysis in higher dimensions are scatter diagrams and parallel coordinates [19, 20]. The former uses an array of scatter diagrams for each pair of objectives. The latter plots an $m$-dimensional objective vector in a two-dimensional graph. Each dimension of the original data is translated to an $x$-coordinate in the two-dimensional plot. This is a very compact way of presenting multidimensional information, but with large sets of data it loses clarity and the analysis becomes more difficult for the DM [2]. Recently, hybrid tools merging parallel coordinates, dendrograms, and cluster maps have been proposed [4].

Recently, level diagrams (LD) [2] have been provided as a practical solution for achieving accurate analysis on $m$-dimensional Pareto fronts by enabling the DM to correlate tendencies between the Pareto set and Pareto front. This visualization has been successfully used in control systems [36, 37, 38, 15], safety systems analysis [52], and reliability engineering [50, 51]. As pointed in [41], LD is one of the most useful methods for visualizing $m$-dimensional Pareto fronts.

A further analysis on objective tradeoff by the DM could include the selection and com- 
parison of various design concepts (i.e. different methods) for solving an MOP. However, the higher the dimension of the objective vector, the greater the difficulty in performing an analysis. This task will be more complex if the aim is to perform a comparison of two (or more) design concepts.

An analysis of the objective exchange when different design concepts are used will provide a better insight into the problem at hand. This new analysis will help the DM compare different design approaches, and evaluate the circumstances where he/she would prefer one over another; furthermore, the DM can decide if the use of a complex concept is justified over a simple concept. In this paper, the LD capabilities are improved by defining a new quality measure to perform an analysis between Pareto fronts (design concepts) for a given MOP statement. This work is developed on two assumptions:

- For the DM it is important to compare the degree of improvement of one design concept over other(s). This could be justified by the fact that some of the qualitative preferences of one design concept are important to bear in mind during the final selection. If there are no preferences for the design concepts under consideration, a global Pareto front could be calculated with solutions from all design concepts; in such case, the analysis on a single Pareto front described in [2] with LD visualization would be enough.

- This visualization is complementary, i.e. it does not substitute the LD visualization technique shown in [2], but it gives additional information to the DM.

The remainder of this paper is as follows: in Section 2 some preliminaries in MOO and design concepts definition are stated. In Section 3 the new framework for concept comparison by means of LD is defined. In Section 4, the new LD framework is validated as a practical tool for concept comparison with a set of MOP. Finally, some concluding remarks are given.

\section{Design concepts preliminaries}

A MOO problem, without loss of generality 11 can be stated as follows:

$$
\min _{\boldsymbol{\theta} \in \Re^{n}} \boldsymbol{J}(\boldsymbol{\theta})=\left[J_{1}(\boldsymbol{\theta}), \ldots, J_{m}(\boldsymbol{\theta})\right] \in \Re^{m}
$$

subject to:

$$
\begin{aligned}
\boldsymbol{g}(\boldsymbol{\theta}) & \leq 0 \\
\boldsymbol{h}(\boldsymbol{\theta}) & =0 \\
\underline{\theta_{i}} \leq \theta_{i} & \leq \overline{\theta_{i}}, i=[1, \ldots, n]
\end{aligned}
$$

\footnotetext{
${ }^{1} \mathrm{~A}$ maximization problem can be converted to a minimization problem. For each of the objectives that has to be maximized, the transformation: $\max J_{i}(\boldsymbol{\theta})=-\min \left(-J_{i}(\boldsymbol{\theta})\right)$ could be applied.
} 
where $\boldsymbol{\theta} \in \Re^{n}$ is defined as the decision vector, $\boldsymbol{J}(\boldsymbol{\theta})$ as the objective vector, and $\boldsymbol{g}(\boldsymbol{\theta})$, $\boldsymbol{h}(\boldsymbol{\theta})$ as the inequality and equality constraint vectors respectively; $\underline{\theta_{i}}, \overline{\theta_{i}}$ are the lower and upper bounds in the decision space.

Usually, there is no single solution because no solution is better in all objectives. Therefore, a set of solutions, the Pareto set, is defined. Each solution in the Pareto set defines an objective vector in the Pareto front. Each point in the Pareto front is said to be a Pareto optimal solution:

Definition 1. (Pareto optimality [31]): an objective vector $\boldsymbol{J}\left(\boldsymbol{\theta}^{1}\right)$ is Pareto optimal if there is not another objective vector $\boldsymbol{J}\left(\boldsymbol{\theta}^{2}\right)$ such that $J_{i}\left(\boldsymbol{\theta}^{2}\right) \leq J_{i}\left(\boldsymbol{\theta}^{1}\right)$ for all $i \in[1,2, \ldots, m]$ and $J_{j}\left(\boldsymbol{\theta}^{2}\right)<J_{j}\left(\boldsymbol{\theta}^{1}\right)$ for at least one $j, j \in[1,2, \ldots, m]$.

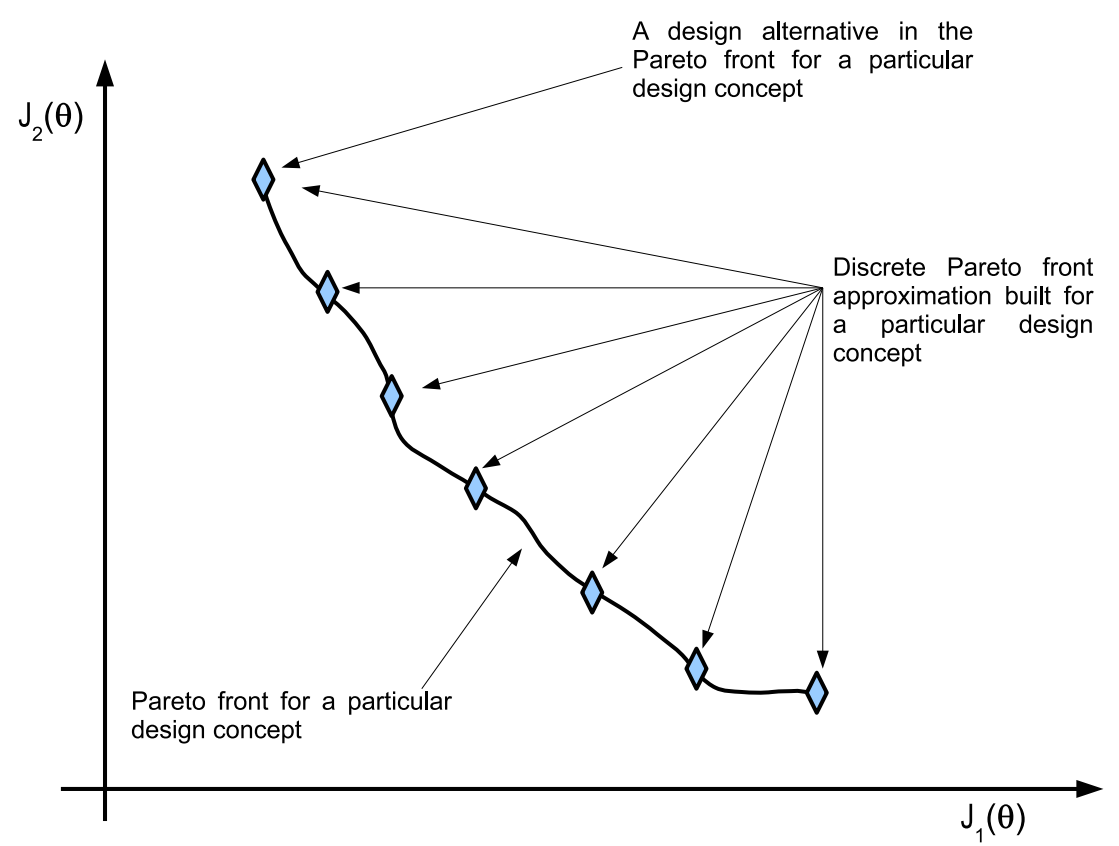

Figure 1: Design concept and design alternative.

A Pareto front is calculated for a given design concept (a design concept is an idea about how to solve a given MOP); this design concept is built with a family of design alternatives, that is, a specific solution in the design concept (see Figure 1). But sometimes, there is a set of different concepts, all of which can solve the MOP. Therefore, the DM can calculate a Pareto front approximation for each. Accordingly, in [28] the definition of the Pareto front and Pareto optimality were extended to a Pareto front for a set of concepts (s-Pareto front) where all solutions are s-Pareto optimal. That is, the s-Pareto front is built with the design alternatives of a set of $K$ design concepts (see Figure 2). 

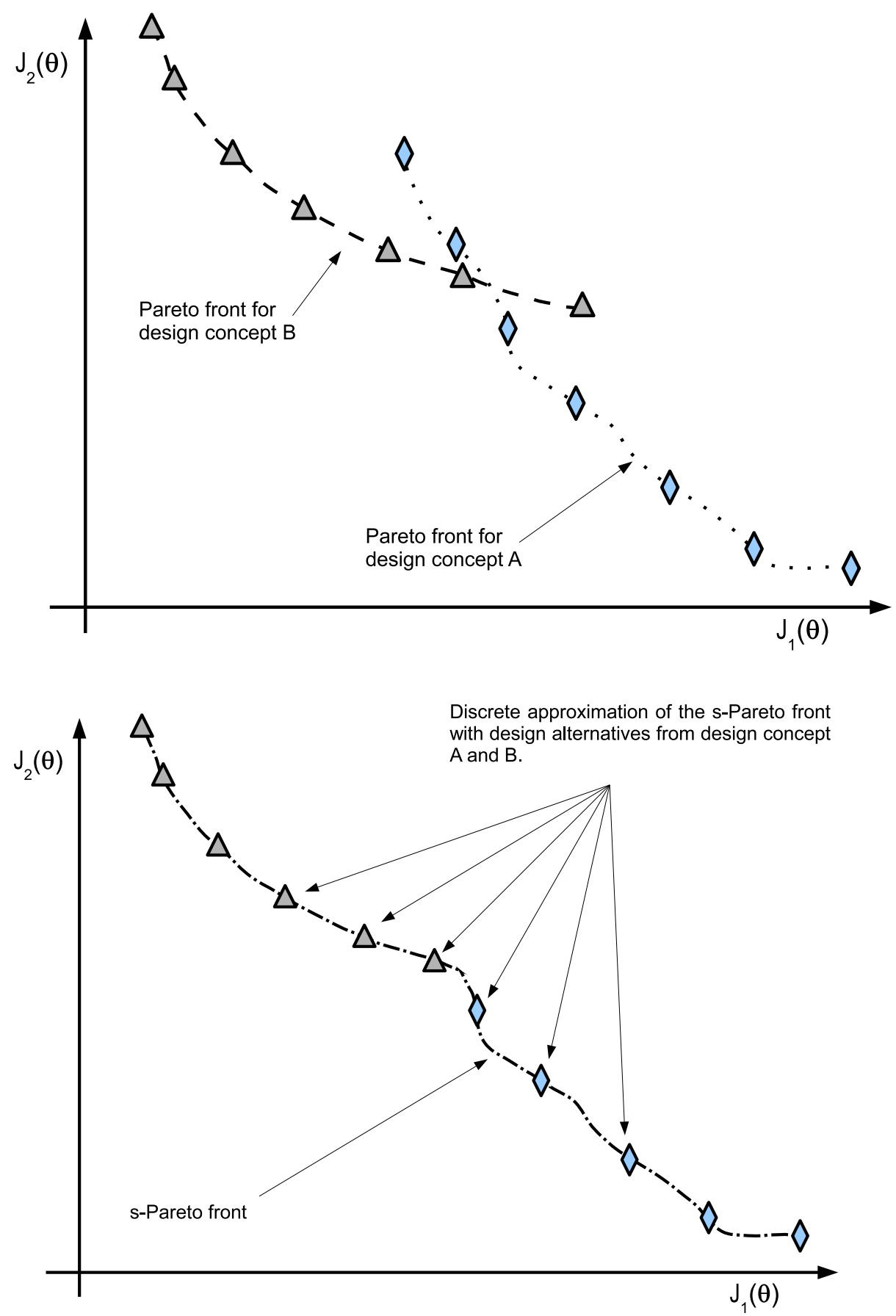

Figure 2: s-Pareto front concept. 
Definition 2. (s-Pareto optimality [28]): an objective vector $\boldsymbol{J}\left(\boldsymbol{\theta}^{1}\right)$ is s-Pareto optimal if there is no other objective vector $\boldsymbol{J}\left(\boldsymbol{\theta}^{2}\right)$ in the design concept $k$ such that $J_{i}\left(\boldsymbol{\theta}^{2}\right) \leq J_{i}\left(\boldsymbol{\theta}^{1}\right)$ for all $i \in[1,2, \ldots, m]$ and all concepts $k, k \in[1, \ldots, K]$; and $J_{j}\left(\boldsymbol{\theta}^{2}\right)<J_{j}\left(\boldsymbol{\theta}^{1}\right)$ for at least one $j, j \in[1,2, \ldots, m]$ for any concept $k$.

A comparison between design concepts is useful for the designer, because he will be able to identify concept strengths, weaknesses, limitations, and drawbacks 28]. It is important to visualize such comparisons, and to have a quantitative measure to evaluate such strengths and weaknesses. As LD has been shown to be useful to visualize $m$-dimensional Pareto fronts, we focus this work on the extension of design concept comparison with LD with a new quality measurement to help in evaluation.

\section{Level diagrams for concept comparison}

We propose a new measurement to evaluate design concepts using LD visualization [2]. Firstly, a brief review of LD is presented.

\subsection{Level diagram review}

The LD helps us to perform an analysis on the calculated Pareto front $\boldsymbol{J}_{P}^{*}$. LD visualization is based on the classification of the Pareto front $\boldsymbol{J}_{P}^{*}$. Each objective $J_{q}(\boldsymbol{\theta})$ is normalized with respect to its minimum and maximum values. That is:

$$
\hat{J}_{q}(\boldsymbol{\theta})=\frac{J_{q}(\boldsymbol{\theta})-J_{q}^{\min }}{J_{q}^{\max }-J_{q}^{\min }}, q \in[1, \ldots, m] .
$$

where (with a little abuse of notation):

$$
\begin{aligned}
\boldsymbol{J}^{\text {min }} & =\left[\min _{J_{1}(\boldsymbol{\theta}) \in J_{P}^{*}} J_{1}(\boldsymbol{\theta}), \ldots, \min _{J_{m}(\boldsymbol{\theta}) \in J_{P}^{*}} J_{m}(\boldsymbol{\theta})\right] \\
\boldsymbol{J}^{\text {max }} & =\left[\max _{J_{1}(\boldsymbol{\theta}) \in J_{P}^{*}} J_{1}(\boldsymbol{\theta}), \ldots, \max _{J_{m}(\boldsymbol{\theta}) \in J_{P}^{*}} J_{m}(\boldsymbol{\theta})\right]
\end{aligned}
$$

For each normalized objective vector $\hat{\boldsymbol{J}}(\boldsymbol{\theta})=\left[\hat{J}_{1}(\boldsymbol{\theta}), \ldots, \hat{J}_{m}(\boldsymbol{\theta})\right]$ a p-norm $\|\hat{\boldsymbol{J}}(\boldsymbol{\theta})\|_{p}$ is applied to evaluate the distance to an ideal solution $\boldsymbol{J}^{\text {ideal }}=\boldsymbol{J}^{\text {min }}$; common norms are:

$$
\begin{aligned}
\|\hat{\boldsymbol{J}}(\boldsymbol{\theta})\|_{1} & =\sum_{q=1}^{m} \hat{J}_{q}(\boldsymbol{\theta}) \\
\|\hat{\boldsymbol{J}}(\boldsymbol{\theta})\|_{2} & =\sum_{q=1}^{m} \hat{J}_{q}(\boldsymbol{\theta})^{2} \\
\|\hat{\boldsymbol{J}}(\boldsymbol{\theta})\|_{\infty} & =\max \hat{J}_{q}(\boldsymbol{\theta})
\end{aligned}
$$




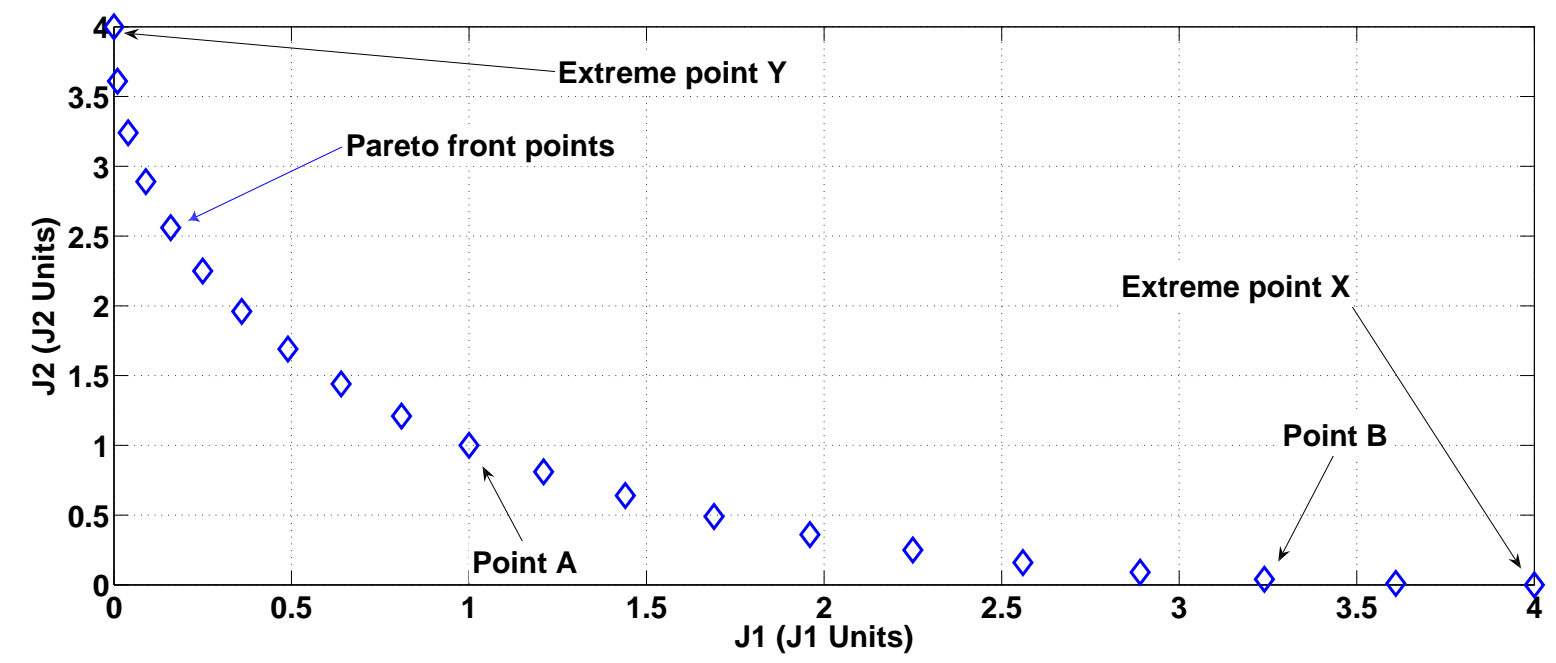

(a)
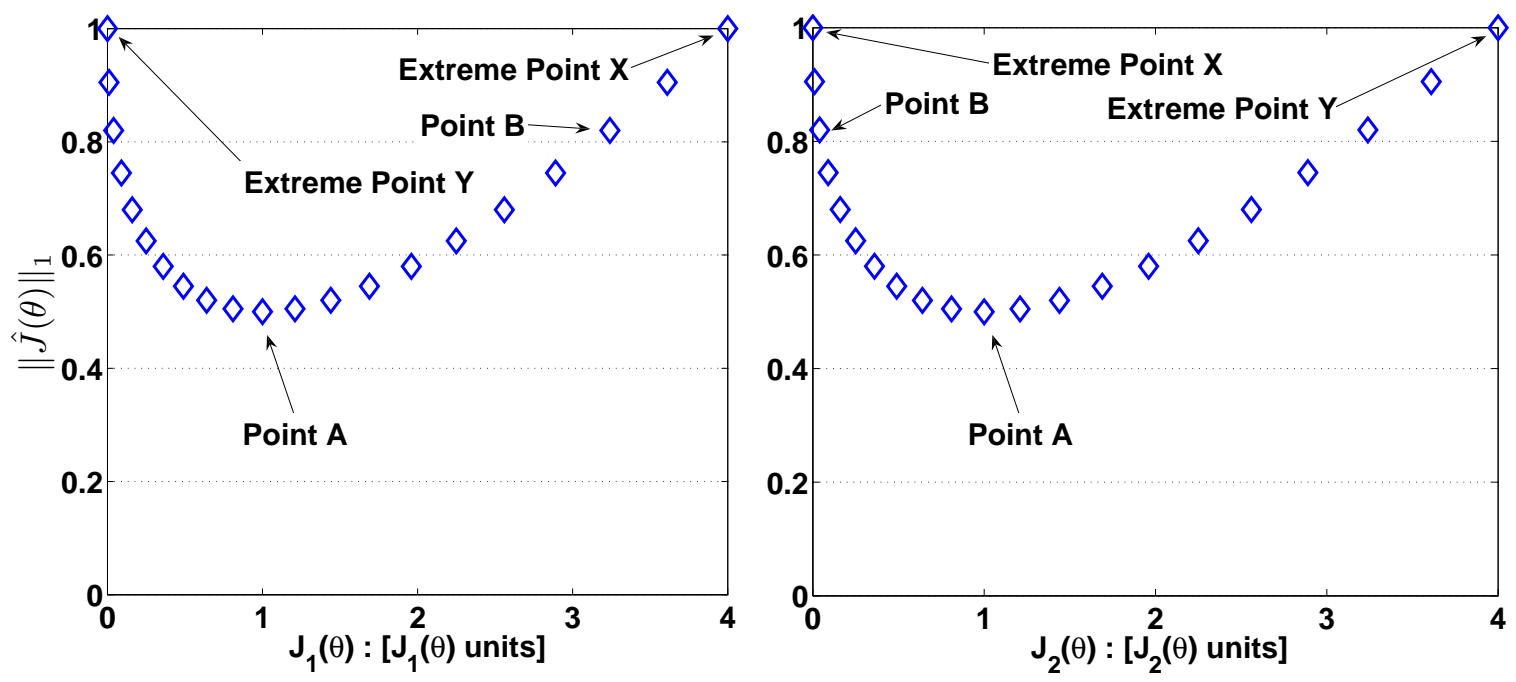

(b)

Figure 3: Typical representation of the Pareto front for bi-objective problems using 2-D graph (a) and LD (b). Points at the same level in LD correspond on each graphic. 
The LD visualization displays a two-dimensional graph for every objective and every decision variable. The ordered pairs $\left(J_{q}(\boldsymbol{\theta}),\|\hat{\boldsymbol{J}}(\boldsymbol{\theta})\|_{p}\right)$ are plotted in each objective subgraph and $\left(\theta_{l},\|\hat{\boldsymbol{J}}(\boldsymbol{\theta})\|_{p}\right)$ in each decision variable sub-graph. Therefore, a given solution will have the same $y$-value in all graphs (see Figure 3). This correspondence helps to evaluate general tendencies along the Pareto front and compare solutions according to the selected norm. In all cases, the lower the norm, the closer the ideal solution 2 . For the remainder of this work and for sake of simplicity, $\|\cdot\|_{2}$ norm will be used.

Differences in LD from other visualization techniques rely mainly on the number of plots required to depict all the information (completeness). For example, scatter plots (SCp) require at least $\frac{m(m-1)}{2}$ graphs for trade-off comparison of each pair of objectives; if correlations with the decision space are needed, $n \cdot m$ additional plots will be required. Another popular visualization strategy is Parallel Coordinates $\left(\|_{p}\right)$, which is a very compact way to display multidimensional data. A single 2-dimensional plot is required, with as many vertical axes as objectives (plus decision variables, if required). Nevertheless, to fully appreciate all the tradeoff among objectives, some re-arrangements of the vertical axes could be required. In the case of LD, a total of $n+m$ graphs are needed and no additional rearrangement for axis is required. Furthermore, the decision space variables inherit the norm value from the objective space, giving a straightforward correlation between objective and decision space. The $\mathrm{LD}$ visualization would offer a tradeoff between $\mathrm{SCp}$ and $\|_{p}$ to visualize an m-dimensional Pareto front if we make the following assumption: the fewer the plots required to depict all the information, the greater the persistence.

In any case, some degree of interactivity (with the data) would be desirable to facilitate the decision making procedure; this could improve the simplicity of any visualization technique. Moreover, elementary tools to identify preferable zones could be helpful (such as color coding for example); this could improve the persistence of the aforementioned visualization techniques. These types of features have been successfully included in software tools such as VIDEO [23] (classical visualization) and TULIP (parallel coordinates) from INRIA 3 .

The LD-ToolBox 4, a powerful tool to analyze $m$-objective Pareto fronts, [36, 38, 52] uses $\mathrm{LD}$ visualization and is employed at this point. It is a Matlab (c) toolbox that offers to the DM a degree of interactivity with multidimensional data. An in-depth explanation of LD tool capabilities can be found in [2]. In [37], an initial approach to compare design concepts with LD was presented. Nevertheless, this analysis is difficult, because it is not possible to have a quantitative measurement to decide which concept performs best. As it is difficult to obtain such an insight with current norms, a new measurement is required for this purpose.

\footnotetext{
${ }^{2}$ In this paper, the minimal values for each objective in the calculated Pareto front approximation are used to build an ideal solution.

${ }^{3}$ Available at http://tulip.labri.fr/TulipDrupal/

${ }^{4}$ available at http://www . mathworks . com/matlabcentral/fileexchange/24042
} 


\begin{tabular}{ccl}
\hline \hline$I_{\epsilon}\left(\boldsymbol{J}_{p 1}^{*}, \boldsymbol{J}_{p 2}^{*}\right)<1$ & $\rightarrow$ & Every $\boldsymbol{J}^{2}\left(\boldsymbol{\theta}^{\mathbf{2}}\right) \in \quad \boldsymbol{J}_{p 2}^{*}$ is \\
& strictly dominated by at \\
& least one $\boldsymbol{J}^{1}\left(\boldsymbol{\theta}^{\mathbf{1}}\right) \in \boldsymbol{J}_{p 1}^{*}$. \\
\hline$I_{\epsilon}\left(\boldsymbol{J}_{p 1}^{*}, \boldsymbol{J}_{p 2}^{*}\right)=1 \wedge I_{\epsilon}\left(\boldsymbol{J}_{p 2}^{*}, \boldsymbol{J}_{p 1}^{*}\right)=1 \rightarrow$ & $\boldsymbol{J}_{p 1}^{*}=\boldsymbol{J}_{p 2}^{*}$. \\
\hline$I_{\epsilon}\left(\boldsymbol{J}_{p 1}^{*}, \boldsymbol{J}_{p 2}^{*}\right)>1 \wedge I_{\epsilon}\left(\boldsymbol{J}_{p 2}^{*}, \boldsymbol{J}_{p 1}^{*}\right)>1 \rightarrow$ & Neither $\boldsymbol{J}_{p 1}^{*}$ weakly domi- \\
& nates $\boldsymbol{J}_{p 2}^{*}$ nor $\boldsymbol{J}_{p 2}^{*}$ weakly \\
& dominates $\boldsymbol{J}_{p 1}^{*}$. \\
\hline
\end{tabular}

Table 1: Interpretations for the $I_{\epsilon}$ indicator.

\subsection{Quality measure to compare design concepts}

As pointed in [28], when multiple design concepts are evaluated by means of their Pareto fronts, it is necessary to have a measurement to quantify their weaknesses and strengths. Both are essential to bring the usefulness of Pareto fronts to conceptual design evaluation.

Several measurements have been developed to evaluate the Pareto front approximations. Nevertheless, many are incompatible or incomplete [53] with the objective vector relations (for example, strict dominance 5 dominance 6 and weak dominance.75)

To evaluate the relative performance between design concepts, the $I_{\epsilon}$ binary indicator [53, 22] is used. This indicator shows the factor $I_{\epsilon}\left(\boldsymbol{J}_{p 1}^{*}, \boldsymbol{J}_{p 2}^{*}\right)$ by which an approximation set $\boldsymbol{J}_{p 1}^{*}$ is worse than another set $\boldsymbol{J}_{p 2}^{*}$ with respect to all the objectives. As detailed in [53], this indicator is complete and compatible, and is useful to determine if two Pareto fronts are incomparable, equal, or if one is better than the other (see Table 1).

Definition 3. The binary $\epsilon$-indicator $I_{\epsilon}\left(\boldsymbol{J}_{p 1}^{*}, \boldsymbol{J}_{p 2}^{*}\right)[5]$ for two Pareto front approximations $\boldsymbol{J}_{p 1}^{*}, \boldsymbol{J}_{p 2}^{*}$ is defined as:

$$
I_{\epsilon}\left(\boldsymbol{J}_{p 1}^{*}, \boldsymbol{J}_{p 2}^{*}\right)=\max _{\boldsymbol{J}^{2}\left(\boldsymbol{\theta}^{2}\right) \in \boldsymbol{J}_{p 2}^{*}} \epsilon_{\boldsymbol{J}^{2}\left(\boldsymbol{\theta}^{2}\right)}
$$

where

$$
\begin{gathered}
\epsilon_{\boldsymbol{J}^{2}\left(\boldsymbol{\theta}^{2}\right)}=\min _{\boldsymbol{J}^{1}\left(\boldsymbol{\theta}^{1}\right) \in \boldsymbol{J}_{p 1}^{*}} \epsilon_{\boldsymbol{J}^{1}\left(\boldsymbol{\theta}^{1}\right), \boldsymbol{J}^{2}\left(\boldsymbol{\theta}^{2}\right)} \\
\epsilon_{\boldsymbol{J}^{1}\left(\boldsymbol{\theta}^{1}\right), \boldsymbol{J}^{2}\left(\boldsymbol{\theta}^{2}\right)}=\max _{1 \leq l \leq m} \frac{J^{1}\left(\boldsymbol{\theta}^{1}\right)_{l}}{J^{2}\left(\boldsymbol{\theta}^{2}\right)_{l}}, \forall \boldsymbol{J}^{1}\left(\boldsymbol{\theta}^{1}\right) \in \boldsymbol{J}_{p 1}^{*}, \boldsymbol{J}^{2}\left(\boldsymbol{\theta}^{2}\right) \in \boldsymbol{J}_{p 2}^{*}
\end{gathered}
$$

\footnotetext{
${ }^{5}$ An objective vector $\boldsymbol{J}\left(\boldsymbol{\theta}^{1}\right)$ strictly dominates another vector $\boldsymbol{J}\left(\boldsymbol{\theta}^{2}\right)$ if $\boldsymbol{J}\left(\boldsymbol{\theta}^{1}\right)$ is better than $\boldsymbol{J}\left(\boldsymbol{\theta}^{2}\right)$ in all objectives.

${ }^{6}$ An objective vector $\boldsymbol{J}\left(\boldsymbol{\theta}^{1}\right)$ dominates another vector $\boldsymbol{J}\left(\boldsymbol{\theta}^{2}\right)$ if $\boldsymbol{J}\left(\boldsymbol{\theta}^{1}\right)$ is not worse than $\boldsymbol{J}\left(\boldsymbol{\theta}^{2}\right)$ in all objectives and is better in at least one objective.

${ }^{7}$ An objective vector $\boldsymbol{J}\left(\boldsymbol{\theta}^{1}\right)$ weakly dominates another vector $\boldsymbol{J}\left(\boldsymbol{\theta}^{2}\right)$ if $\boldsymbol{J}\left(\boldsymbol{\theta}^{1}\right)$ is not worse than $\boldsymbol{J}\left(\boldsymbol{\theta}^{2}\right)$ in all objectives.
} 


\begin{tabular}{|c|c|c|c|}
\hline 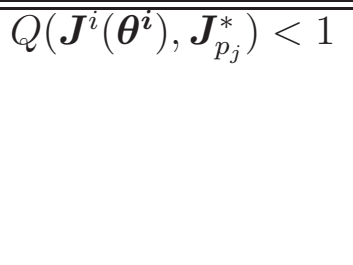 & $\rightarrow$ & $\begin{array}{l}\boldsymbol{J}^{i}\left(\boldsymbol{\theta}^{i}\right) \in \boldsymbol{J}_{p_{i}}^{*} \text { strictly domi- } \\
\text { nates at least one } \boldsymbol{J}^{j}\left(\boldsymbol{\theta}^{j}\right) \in \\
\boldsymbol{J}_{p_{j}}^{*} \text {. }\end{array}$ & $\begin{array}{l}\boldsymbol{J}^{i}\left(\boldsymbol{\theta}^{\mathbf{1}}\right) \in \boldsymbol{J}_{p_{i}}^{*} \text { has an im- } \\
\text { provement over a solution } \\
\boldsymbol{J}^{j}\left(\boldsymbol{\theta}^{\boldsymbol{j}}\right) \in \boldsymbol{J}_{p_{j}}^{*} \text { by a scale } \\
\text { factor of } Q\left(\boldsymbol{J}^{i}\left(\boldsymbol{\theta}^{\boldsymbol{i}}\right), \boldsymbol{J}_{p_{j}}^{*}\right) \text { (at } \\
\text { least) for all objectives. }\end{array}$ \\
\hline$Q\left(\boldsymbol{J}^{i}\left(\boldsymbol{\theta}^{\boldsymbol{i}}\right), \boldsymbol{J}_{p_{j}}^{*}\right)=1$ & $\rightarrow$ & $\begin{array}{l}\boldsymbol{J}^{i}\left(\boldsymbol{\theta}^{\boldsymbol{i}}\right) \in \boldsymbol{J}_{p_{i}}^{*} \text { is not com- } \\
\text { parable with any solution } \\
\boldsymbol{J}^{j}\left(\boldsymbol{\theta}^{\boldsymbol{j}}\right) \in \boldsymbol{J}_{p_{j}}^{*} \text {. }\end{array}$ & $\begin{array}{l}\boldsymbol{J}^{i}\left(\boldsymbol{\theta}^{\boldsymbol{i}}\right) \in \boldsymbol{J}_{p_{i}}^{*} \text { is Pareto opti- } \\
\text { mal in } \boldsymbol{J}_{p_{j}}^{*} \text { or } \boldsymbol{J}^{i}\left(\boldsymbol{\theta}^{\boldsymbol{i}}\right) \in \boldsymbol{J}_{p_{i}}^{*} \\
\text { is inside a region in the ob- } \\
\text { jective space not covered by } \\
\boldsymbol{J}_{p_{j}}^{*} .\end{array}$ \\
\hline$Q\left(\boldsymbol{J}^{i}\left(\boldsymbol{\theta}^{\boldsymbol{i}}\right), \boldsymbol{J}_{p_{j}}^{*}\right)>1$ & $\rightarrow$ & $\begin{array}{l}\boldsymbol{J}^{i}\left(\boldsymbol{\theta}^{\boldsymbol{i}}\right) \in \boldsymbol{J}_{p_{i}}^{*} \text { is strictly } \\
\text { dominated by at least one } \\
\boldsymbol{J}^{j}\left(\boldsymbol{\theta}^{\boldsymbol{j}}\right) \in \boldsymbol{J}_{p_{j}}^{*} .\end{array}$ & $\begin{array}{l}\text { A solution } \boldsymbol{J}^{j}\left(\boldsymbol{\theta}^{\boldsymbol{j}}\right) \in \boldsymbol{J}_{p_{j}}^{*} \\
\text { has an improvement over } \\
\boldsymbol{J}^{i}\left(\boldsymbol{\theta}^{\boldsymbol{i}}\right) \in \boldsymbol{J}_{p_{i}}^{*} \text { by a scale of } \\
Q\left(\boldsymbol{J}^{i}\left(\boldsymbol{\theta}^{i}\right), \boldsymbol{J}_{p_{j}}^{*}\right) \text { (at least) for } \\
\text { all objectives. }\end{array}$ \\
\hline
\end{tabular}

Table 2: Comparison methods using the $Q\left(\boldsymbol{J}^{i}\left(\boldsymbol{\theta}^{i}\right), \boldsymbol{J}_{p_{j}}^{*}\right)$ quality measure and its meaning.

As the binary $\epsilon$-indicator is a scalar measure between Pareto fronts, some modifications are required to build a scalar measure for each design alternative on each concept. The quality indicator $Q\left(\boldsymbol{J}^{i}\left(\boldsymbol{\theta}^{\boldsymbol{i}}\right), \boldsymbol{J}_{p_{j}}^{*}\right) 8$ is defined for this purpose.

Definition 4. The quality indicator $Q\left(\boldsymbol{J}^{i}\left(\boldsymbol{\theta}^{i}\right), \boldsymbol{J}_{p_{j}}^{*}\right)$ for two design concepts $i, j \in[1, \ldots, K]$ is defined as:

$$
Q\left(\boldsymbol{J}^{i}\left(\boldsymbol{\theta}^{i}\right), \boldsymbol{J}_{p_{j}}^{*}\right)=\left\{\begin{array}{cl}
1 & \text { if } \min \epsilon_{\boldsymbol{J}^{i}\left(\boldsymbol{\theta}^{i}\right), \boldsymbol{J}_{p_{j}}^{*}}>1 \wedge \min \epsilon_{\boldsymbol{J}^{j}\left(\boldsymbol{\theta}^{j}\right), \boldsymbol{J}_{p_{i}}^{*}}>1 \\
\min _{\boldsymbol{J}^{j}\left(\boldsymbol{\theta}^{j}\right) \in \boldsymbol{J}_{p j}^{*}} \epsilon_{\boldsymbol{J}^{i}\left(\boldsymbol{\theta}^{i}\right), \boldsymbol{J}_{p_{j}}^{*}} & \text { otherwise }
\end{array}\right.
$$

where:

$$
\epsilon_{\boldsymbol{J}^{i}\left(\boldsymbol{\theta}^{i}\right), \boldsymbol{J}_{p_{j}}^{*}}=\max _{1 \leq l \leq m} \frac{J^{i}\left(\boldsymbol{\theta}^{i}\right)_{l}}{J^{j}\left(\boldsymbol{\theta}^{j}\right)_{l}}, \boldsymbol{J}^{i}\left(\boldsymbol{\theta}^{i}\right) \in \boldsymbol{J}_{p_{i}}^{*}, \forall \boldsymbol{J}^{j}\left(\boldsymbol{\theta}^{j}\right) \in \boldsymbol{J}_{p_{j}}^{*}
$$

with $\boldsymbol{\theta}^{i} \in \boldsymbol{\Theta}_{p_{i}}^{*}, \boldsymbol{J}^{i}\left(\boldsymbol{\theta}^{i}\right) \in \boldsymbol{J}_{p_{i}}^{*}, i \neq j$.

With this quality measurement and the LD we can visualize regions in the Pareto front where a design concept is better or worse than another. Furthermore, we will have a measurement of how much better one design concept performs than the other (see Figure 4).

\footnotetext{
${ }^{8}$ To avoid problems with this quality indicator when the objective vector has positive and negative values or zero values are involved, a normalization in the range $[1,2]$ for each objective is used as a preliminary step.
} 


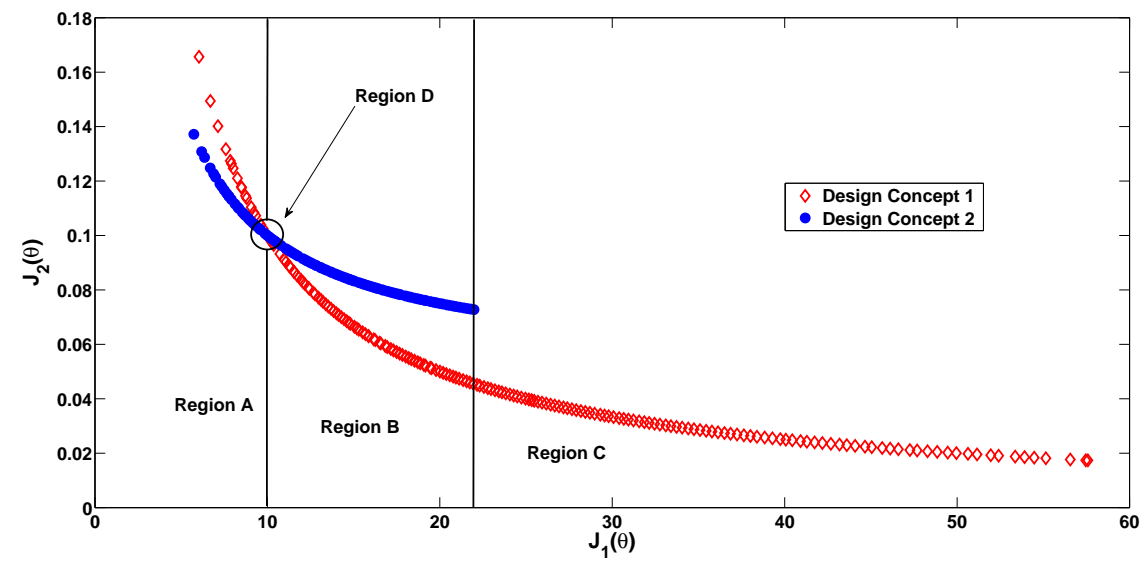

(a)
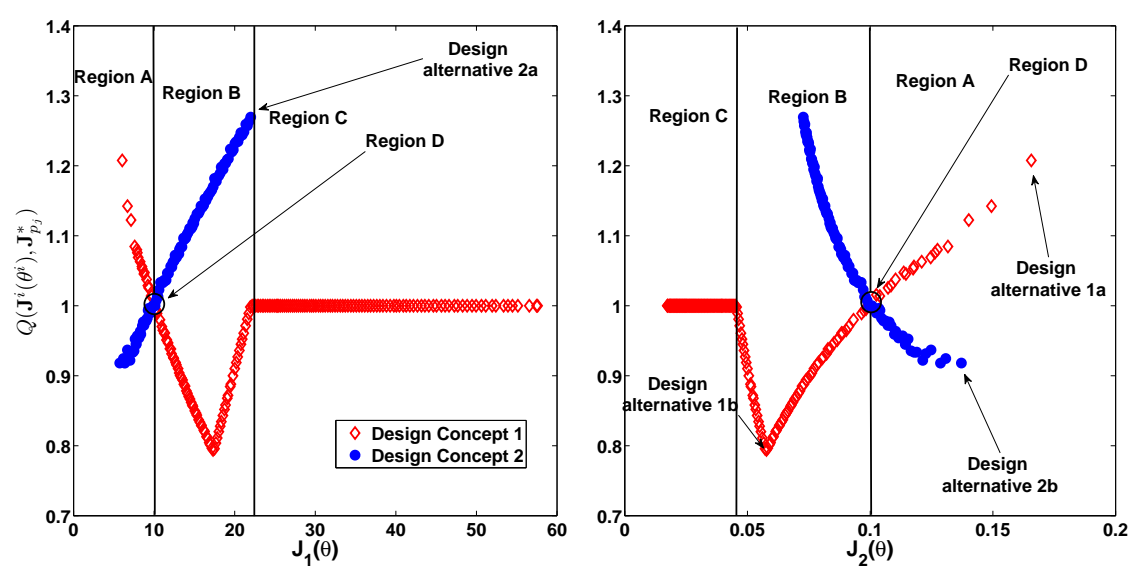

(b)
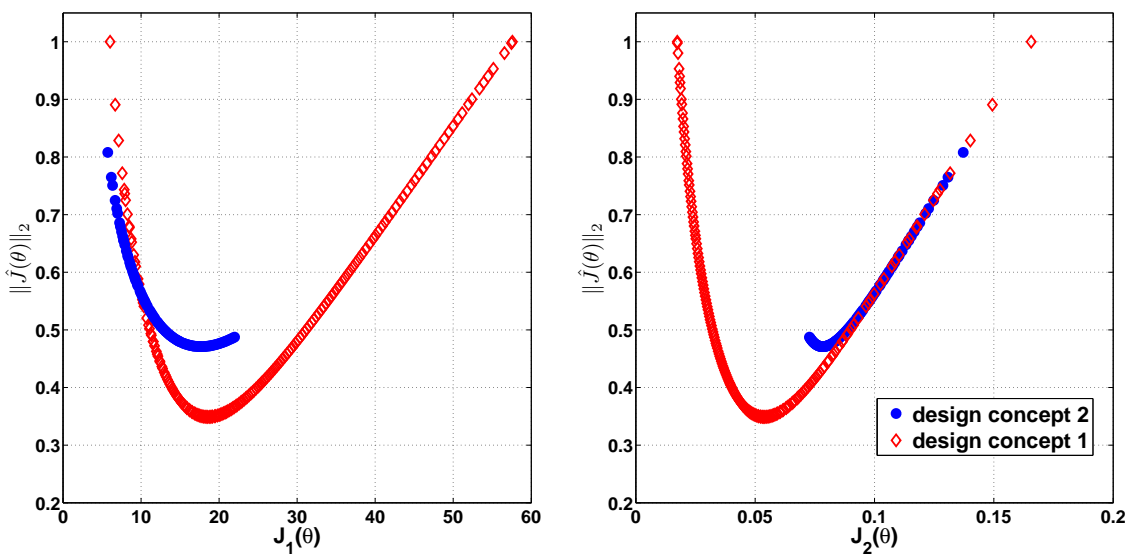

(c)

Figure 4: Typical comparison of two design concepts using a 2-D graph (a), level diagrams with quality measure $Q(\cdot, \cdot)$ (b) and level diagrams with 2-norm (c). 
For example, in Figure 4 the relationships described in Table 2 can be seen. Firstly, due to the quality measurement, it is possible to quickly identify the s-Pareto non-optimal (any solution $Q\left(\boldsymbol{J}^{i}\left(\boldsymbol{\theta}^{\boldsymbol{i}}\right), \boldsymbol{J}_{p_{j}}^{*}\right)>1$ ) from s-Pareto optimal solutions (any solution $Q\left(\boldsymbol{J}^{i}\left(\boldsymbol{\theta}^{\boldsymbol{i}}\right), \boldsymbol{J}_{p_{j}}^{*}\right) \leq$ 1). Moreover, the quality measurement enables us to assign a quantitative value about how better or worse a solution is with respect another concept. Further analysis with this quality measurement can be made for particular solutions, or for regions in the LD.

Regarding particular solutions, let us see for example design alternatives 1a, 2a, 1b and 2b (Figure 4b). We have:

- $Q\left(1 a, \boldsymbol{J}_{p 2}^{*}\right) \approx 1.2$. That is, among the solutions $\boldsymbol{J}^{2}\left(\boldsymbol{\theta}^{2}\right) \in \boldsymbol{J}_{p 2}^{*}$ which dominate objective vector 1 a, the smaller $k$ for a solution $\boldsymbol{J}^{2}\left(\boldsymbol{\theta}^{2}\right)$ such that $\boldsymbol{J}^{2^{\prime}}\left(\boldsymbol{\theta}^{2}\right)=k \cdot \boldsymbol{J}^{2}\left(\boldsymbol{\theta}^{2}\right)$ is weakly dominated by $1 \mathrm{a}$ is $k \approx 1.2$.

- $Q\left(1 b, \boldsymbol{J}_{p 2}^{*}\right) \approx 0.8$. That is, among the solutions $\boldsymbol{J}^{2}\left(\boldsymbol{\theta}^{2}\right) \in \boldsymbol{J}_{p 2}^{*}$ dominated by objective vector 1 b, the bigger $k$ for a solution $\boldsymbol{J}^{2}\left(\boldsymbol{\theta}^{2}\right)$ such that $\boldsymbol{J}^{2^{\prime}}\left(\boldsymbol{\theta}^{2}\right)=k \cdot \boldsymbol{J}^{2}\left(\boldsymbol{\theta}^{2}\right)$ weakly dominates $1 \mathrm{~b}$ is $k \approx 0.8$.

- $Q\left(2 a, \boldsymbol{J}_{p 1}^{*}\right) \approx 1.28$. That is, among the solutions $\boldsymbol{J}^{1}\left(\boldsymbol{\theta}^{1}\right) \in \boldsymbol{J}_{p 1}^{*}$ which dominate objective vector 2 a, the smaller $k$ for a solution $\boldsymbol{J}^{1}\left(\boldsymbol{\theta}^{1}\right)$ such that $\boldsymbol{J}^{1^{\prime}}\left(\boldsymbol{\theta}^{1}\right)=k \cdot \boldsymbol{J}^{1}\left(\boldsymbol{\theta}^{1}\right)$ is weakly dominated by $2 \mathrm{a}$ is $k \approx 1.28$.

- $Q\left(2 b, \boldsymbol{J}_{p 1}^{*}\right) \approx 0.9$. That is, among the solutions $\boldsymbol{J}^{1}\left(\boldsymbol{\theta}^{1}\right) \in \boldsymbol{J}_{p 1}^{*}$ dominated by objective vector 2 b, the bigger $k$ for a solution $\boldsymbol{J}^{1}\left(\boldsymbol{\theta}^{1}\right)$ such that $\boldsymbol{J}^{1^{\prime}}\left(\boldsymbol{\theta}^{1}\right)=k \cdot \boldsymbol{J}^{1}\left(\boldsymbol{\theta}^{1}\right)$ weakly dominates $2 \mathrm{~b}$ is $k \approx 0.9$.

With regard to tendencies, the following remarks can be made:

- The lower $J_{1}(\boldsymbol{\theta})$, the better the improvement of concept $2(\bigcirc)$ over concept $1(\diamond)$.

- For $J_{2}(\boldsymbol{\theta}) \in[0.055,0.1]$ approximately, the lower $J_{2}(\boldsymbol{\theta})$, the better the improvement of concept $1(\diamond)$ over concept $2(\bigcirc)$.

Regarding zones in Figure $4 \mathrm{~b}$, region A represents the zone where design concept $2(\bigcirc)$ is better than design concept $1(\diamond)$. Note that the design alternatives from concept 2 have a quality measurement $Q\left(\boldsymbol{J}^{2}\left(\boldsymbol{\theta}^{2}\right), \boldsymbol{J}_{p 1}^{*}\right)<1$ and design alternatives from concept 1 have a quality measurement $Q\left(\boldsymbol{J}^{\mathbf{1}}\left(\boldsymbol{\theta}^{1}\right), \boldsymbol{J}_{p 2}^{*}\right)>1$. The opposite is true for region B. Region $\mathrm{C}$ is a zone reached (covered) only by concept 1 (and thus, it is impossible to compare both concepts). Finally, region D represents a region where both concepts have almost the same exchange between objectives. Please note how this analysis is more difficult with standard norms using LD (see Figure 4c).

Finally, note that although it is possible to build a s-Pareto front merging the design alternatives of each concept and analyze its tendencies, it is difficult to measure the improvement of one concept over another. This fact is mainly due to the loss of information after building the s-Pareto front. The LD with the quality measure enables a quantitative 
a-priori analysis between concepts, and it is possible to decide, for example, if the improvement of one of them is justifiable. For instance, the DM could decide that the improvement by a factor of 0.9 for concept 2 is not justifiable, and decide to only retain concept 1 .

Whilst such comparison can be performed by visual inspection in a classical 2D-objective graph (see Figure $4 \mathrm{a}$ ), such a task will be more complex when three or more objectives are considered. Several examples are considered to show the LD performing comparisons on $m$-dimensional Pareto fronts using this quality measurement.

\section{Examples}

In this section, four examples are analyzed, where different concepts are used to obtain different trade-offs between objectives. The following examples show analysis on 2, 3, 5 and 6 dimensions for the objective vector space. The first example is used to show that no information (completeness) or clarity (simplicity) will be lost using the LD instead a 2-D graphical analysis. The latter cases are presented to show the visualization capacity of LD using the quality measurement for concept comparison in $m$-dimensional Pareto fronts. It is assumed that any superfluous objective has been previously removed from the analysis; i.e. we are depicting all the information (concerning the number of objectives) required by the DM.

For all examples, the LD-ToolBox is used to retain the degree of interactivity with the supplied data (in Appendix A, supplementary videos are provided). Finally, the Pareto fronts are calculated using the sp-MODE algorithm [35, 39], which uses differential evolution (DE) 45, 44, 9] algorithm (see Appendix Appendix B for details), but any other algorithm or procedure could be used since we are developing tools for the MCDM step. For the sake of simplicity, the LD will be coded as follows: $L D /$ front/measure. For example, $\mathrm{LD} / \boldsymbol{J}_{p}^{*} /\|\hat{\boldsymbol{J}}(\boldsymbol{\theta})\|_{2}$, means that a visual representation of Pareto front approximation $\boldsymbol{J}_{p}^{*}$ with 2 -norm in LD is presented.

\subsection{Bi-objective truss design problem}

The truss design problem is a classical MOO benchmark statement to test algorithms, as well as decision-making step procedures. The truss parameters proposed in [43] are used with the design concepts stated in [29, 28]. Two objectives are minimized: deflection $\left(J_{1}(\boldsymbol{\theta})\right)$ and total volume $\left(J_{2}(\boldsymbol{\theta})\right)$. Whereas it could be easiest (from a persistence point of view) to use a straightforward approach with the SCp visualization, this example is included and used to cover the following topics:

- Bring a first approach to the LD visualization and design concept comparison with a (probably well known) multi-objective benchmark problem.

- Introduce some properties of the LD visualization.

In Figure 5, the objective exchange for both concepts and its $\operatorname{LD} /\left\{J_{p 1}^{*}, J_{p 2}^{*}\right\} /\|\hat{\boldsymbol{J}}(\boldsymbol{\theta})\|_{2}$ visualization is shown. It is important to notice that the $L D$ visualization by itself is not 


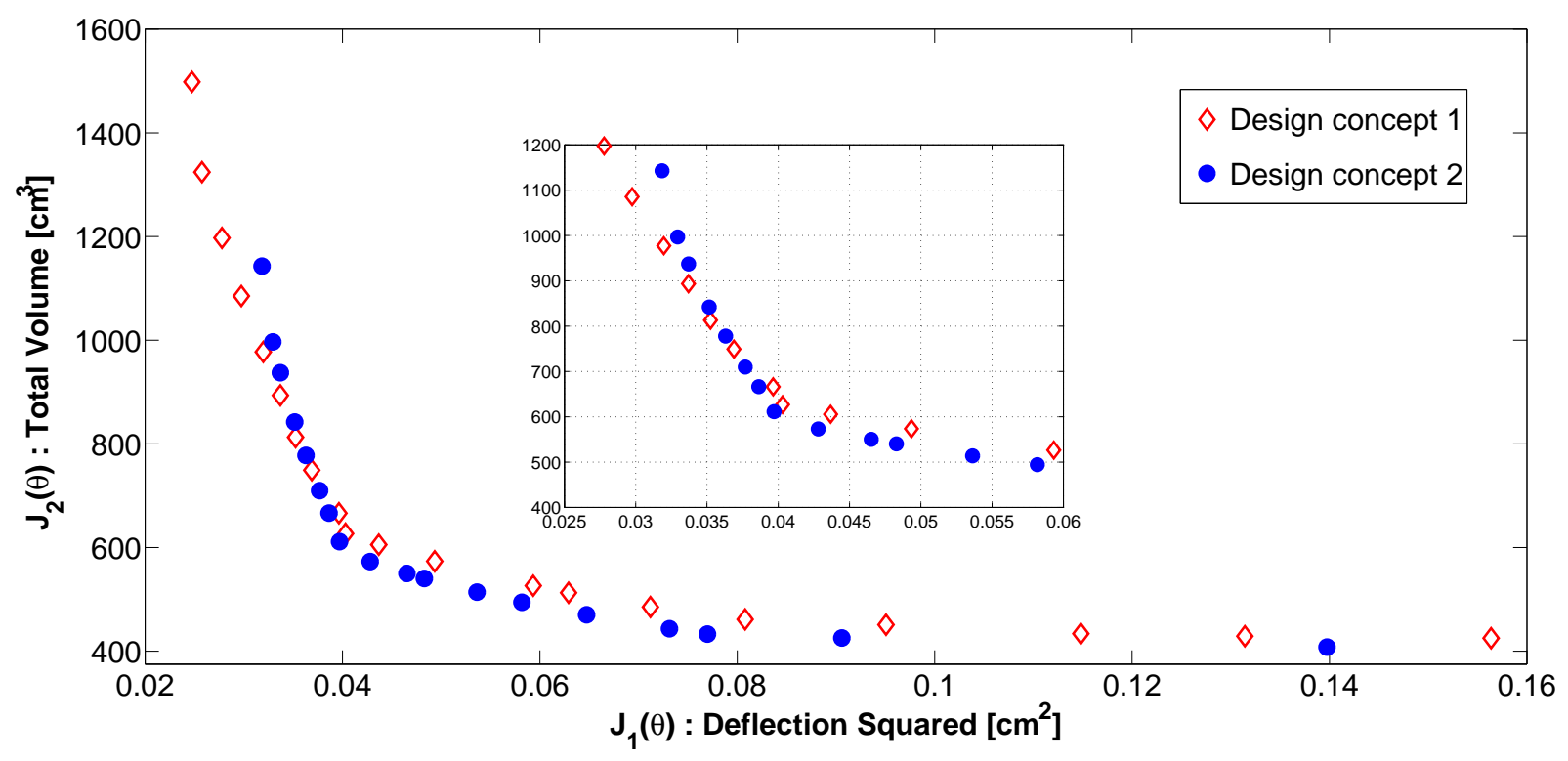

(a)
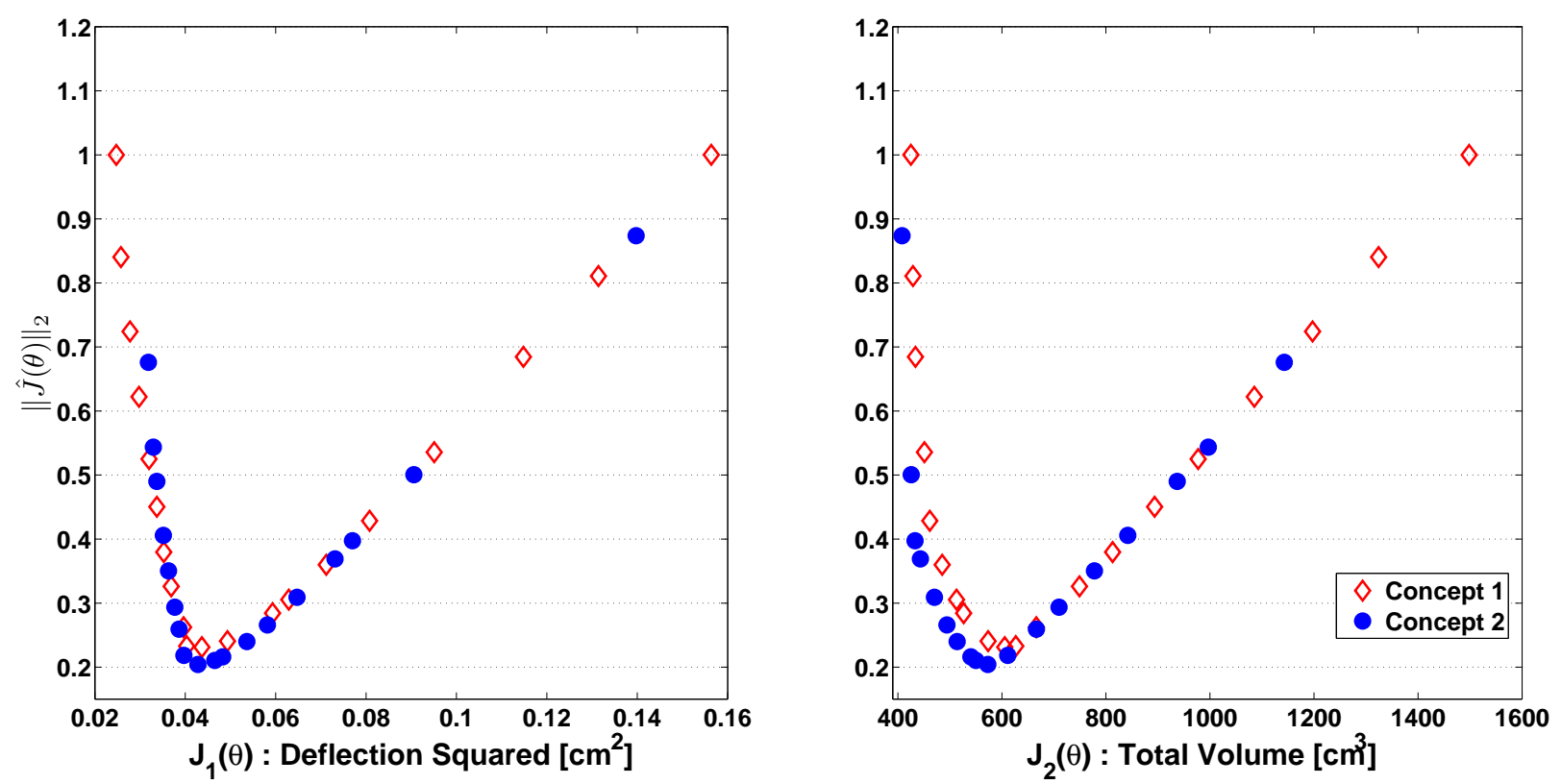

(b)

Figure 5: Objective exchange for two concepts in truss design example (a) and representation using $\mathrm{LD} /\left\{J_{p 1}^{*}, J_{p 2}^{*}\right\} /\|\hat{\boldsymbol{J}}(\boldsymbol{\theta})\|_{2}(\mathrm{~b})$. 
enough to make an analysis or a comparison between design concepts. This is mainly because there is not a quantitative measurement on the improvement of one over the other.

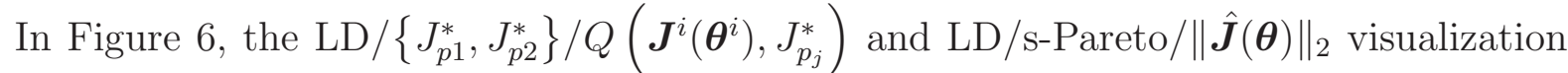
are shown. We can identify in Figure 6a some interesting points:

- In region $\mathrm{B}$, concept $1(\diamond)$ is better than concept $2(\bigcirc)$. The opposite is true for Zone D.

- The lower $J_{2}(\boldsymbol{\theta})$, the better the improvement of concept $2(\bigcirc)$ over concept $1(\diamond)$.

- Region A is covered only by concept $1(\diamond)$.

- In region $\mathrm{C}$, both concepts offer the DM the same type of exchange between objectives.

With this visual inspection, the DM can decide if a $2 \%$ of improvement between concepts is justifiable to change the structural design (assuming that one of the concepts is currently being used). Furthermore, the DM knows that if the concept design requires keeping the deflection $J_{2}(\boldsymbol{\theta}) \in[0.03,0.04]$, then she/he can select, for example, the easier structure to build.

\subsection{Disc brake design}

This is the disc brake design proposed in [33]. The design objectives to be minimized are the mass of the brake $\left(J_{1}(\boldsymbol{\theta})\right)$, the stopping time $\left(J_{2}(\boldsymbol{\theta})\right)$, and the engaging force $\left(J_{3}(\boldsymbol{\theta})\right)$. The decision variables are the inner and outer radius of the disc, the engaging force, and the number of friction surfaces. The latter is used to generate two different design concepts: a disc brake design with 4 and 6 friction surfaces respectively. This example is used to show:

- The complexity increase on visualization when the DM required to analyse more than two objectives.

- To introduce geometrical remarks and their physical meaning to the problem at hand.

In Figure 7 , the objective tradeoff in a 3D-objective plot, and the $\operatorname{LD} /\left\{J_{p 1}^{*}, J_{p 2}^{*}\right\} /\|\hat{\boldsymbol{J}}(\boldsymbol{\theta})\|_{2}$ visualization are presented. It is important to notice the complexity increases from 2 to 3

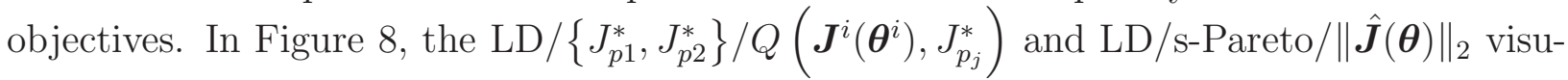
alizations are shown. Some remarks from Figure 8 can be made:

- Both design concepts guarantee a useful coverage on $J_{3}(\boldsymbol{\theta})$.

- No single concept is able to fully cover $J_{1}(\boldsymbol{\theta})$ or $J_{2}(\boldsymbol{\theta})$.

- According to the last observation, if $J_{1}(\boldsymbol{\theta}) \leq 0.5$ is required, concept 1 must be used; however, if $J_{2}(\boldsymbol{\theta}) \leq 4$ is required, concept 2 must be selected.

- The bigger the mass of the brake, the better the improvement of concept $2(\bigcirc)$ over concept $1(\diamond)$ in the range $J_{1}(\boldsymbol{\theta}) \in[0.6,1.0]$. 

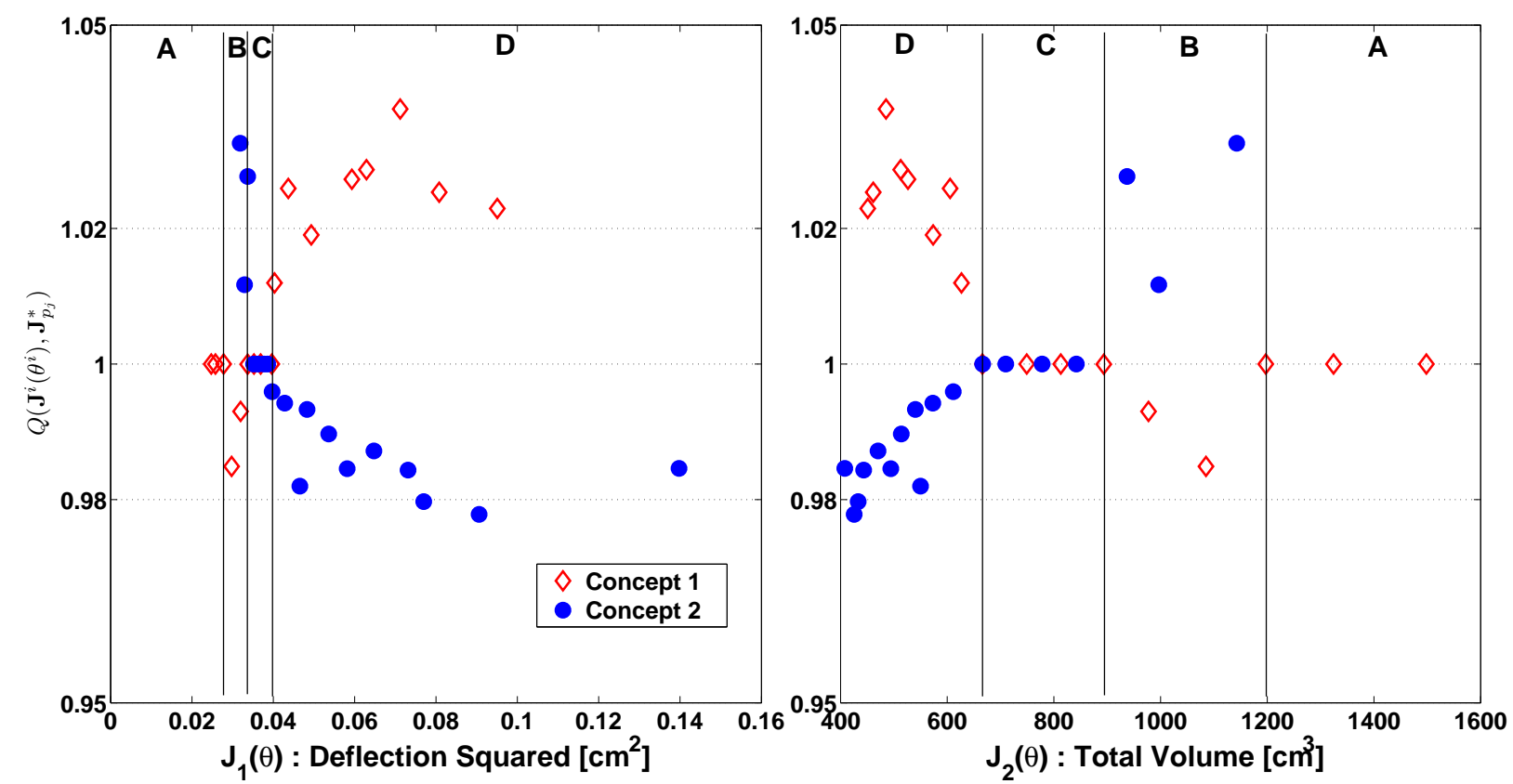

(a)
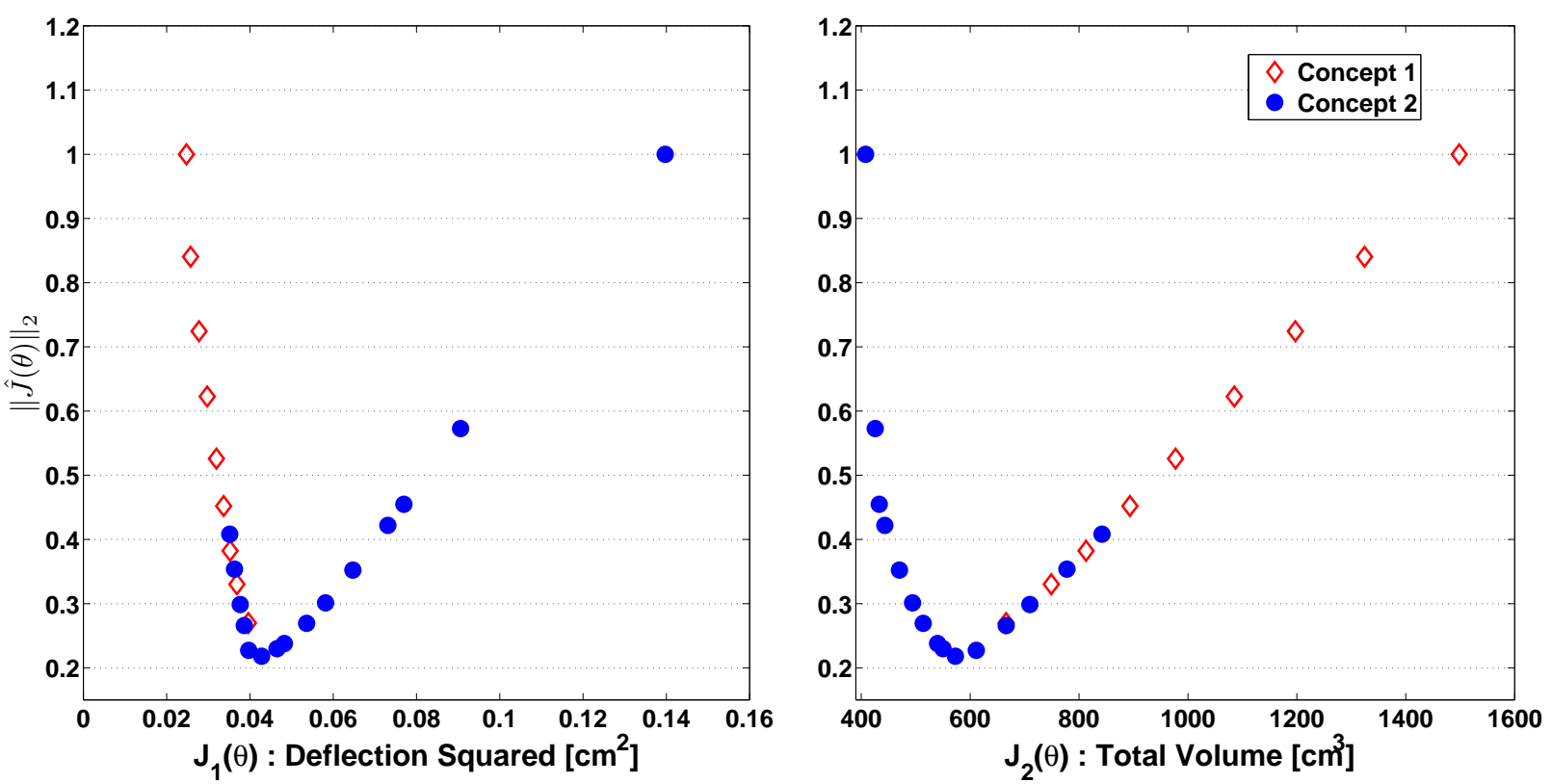

(b)

Figure 6: Concepts comparison for truss design example using $\mathrm{LD} /\left\{J_{p 1}^{*}, J_{p 2}^{*}\right\} / Q\left(\boldsymbol{J}^{1,2}\left(\boldsymbol{\theta}^{1,2}\right), J_{p 2, p 1}^{*}\right)(\mathrm{a})$ and LD/s-Pareto $/\|\hat{\boldsymbol{J}}(\boldsymbol{\theta})\|_{2}$ (b). 


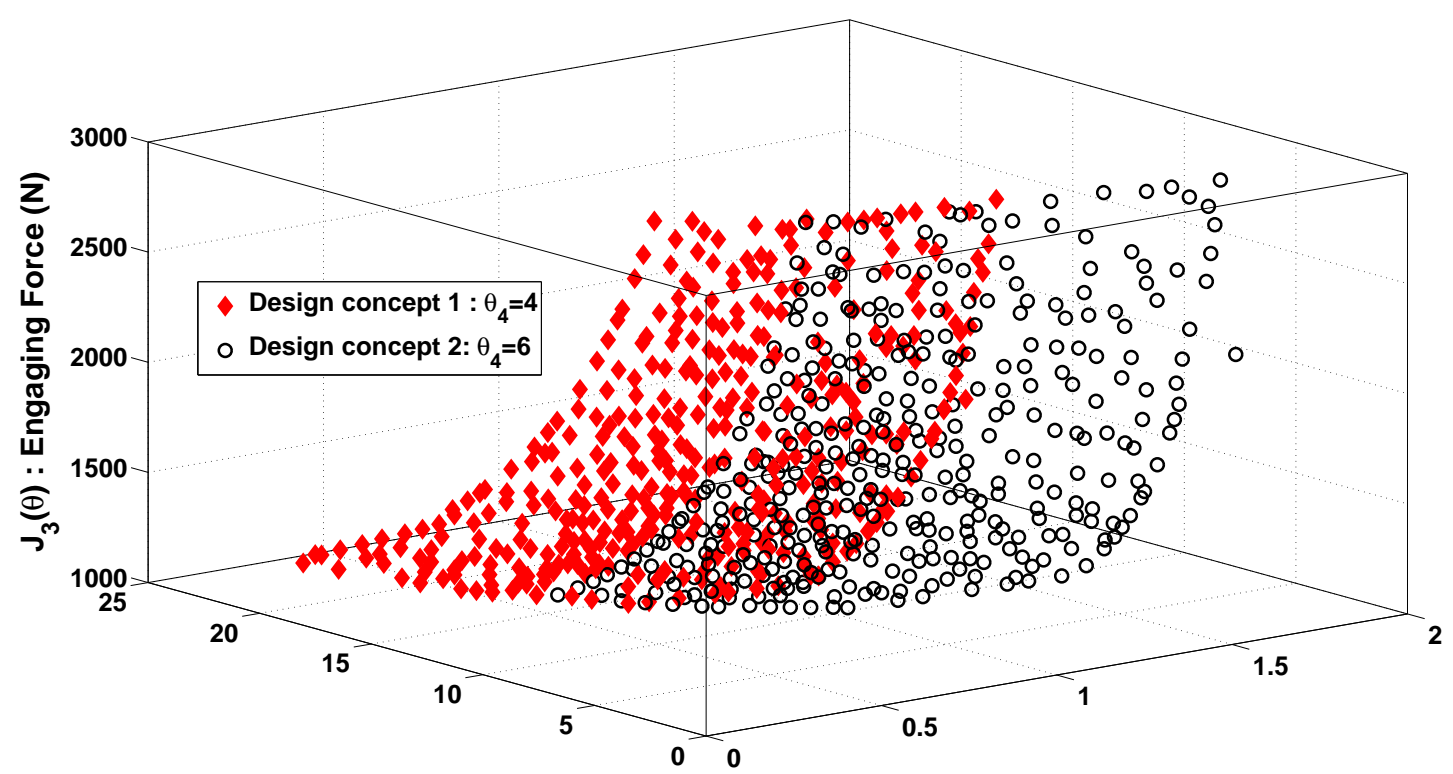

$\mathrm{J}_{2}(\theta)$ : Stopping Time [secs.]

$\mathrm{J}_{1}(\theta)$ : Mass of the brake $[\mathrm{Kg}$.

(a)

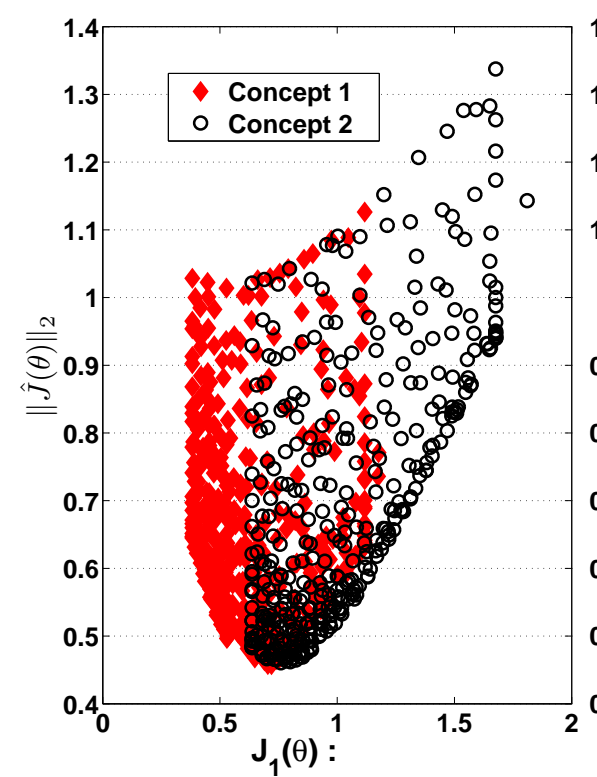

Mass of the brake [Kg.]

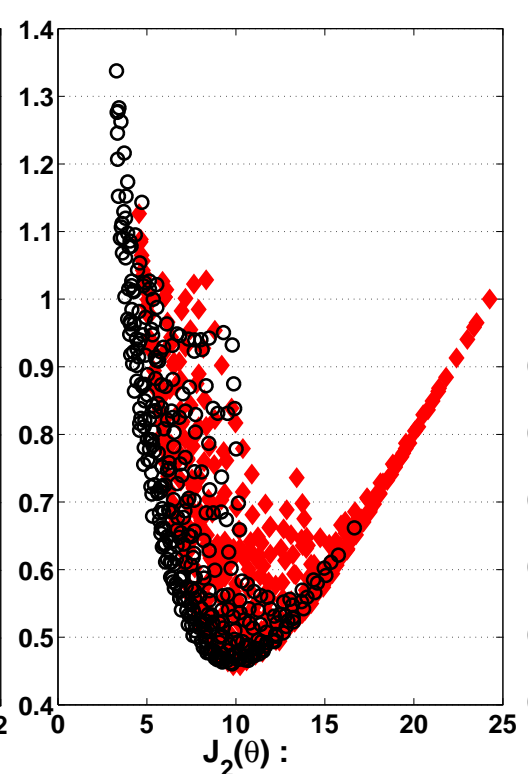

Stopping Time [secs.]

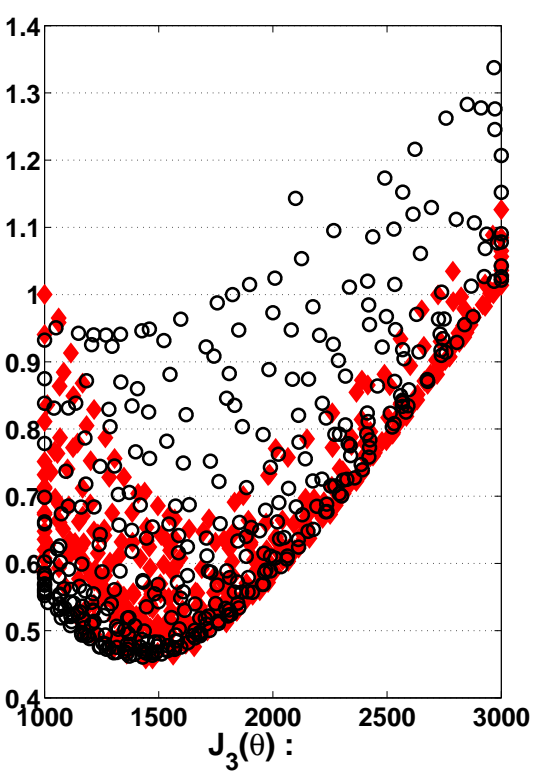

Engaging Force $(\mathrm{N})$

(b)

Figure 7: Objective exchange for two design: concepts in disc brake design example (a) and concepts comparison with $\mathrm{LD} /\left\{J_{p 1}^{*}, J_{p 2}^{*}\right\} /\|\hat{\boldsymbol{J}}(\boldsymbol{\theta})\|_{2}$ (b). 


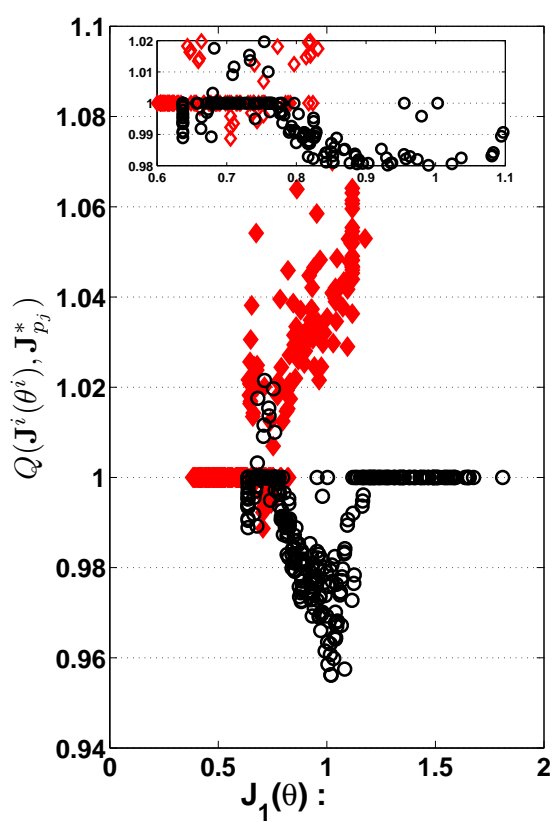

Mass of the brake [Kg.]

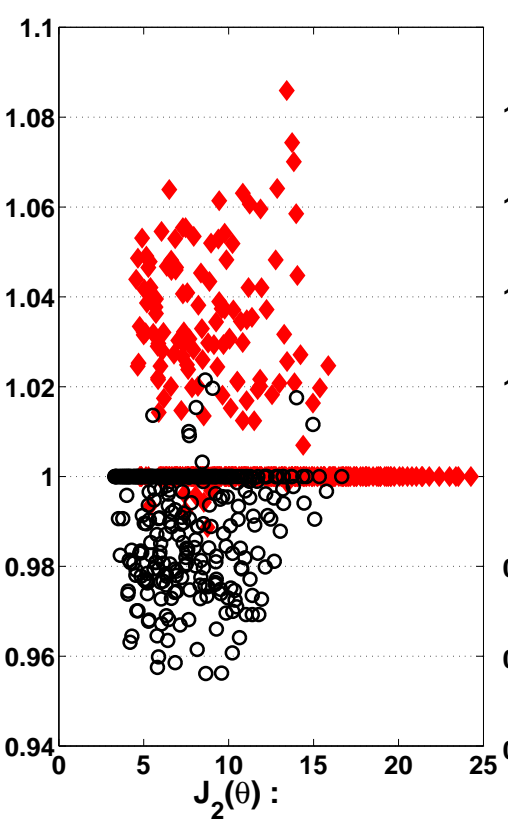

Stopping Time [secs.]

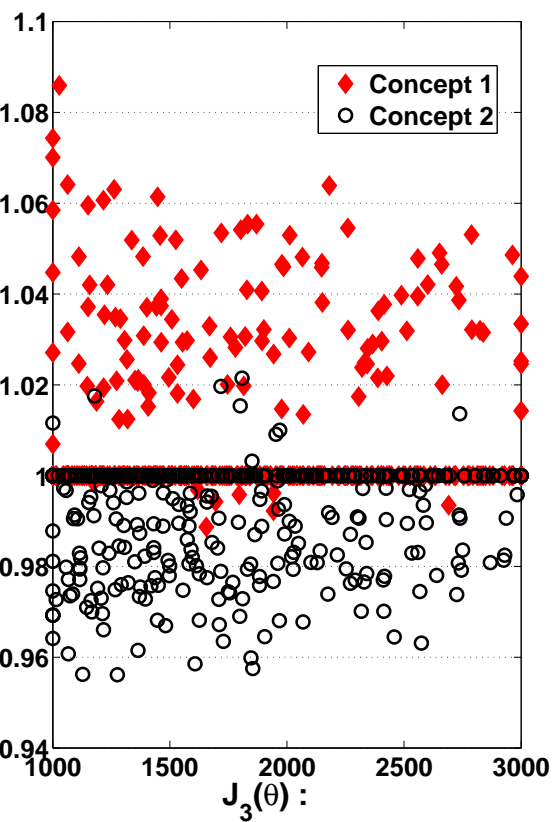

Engaging Force (N)

(a)

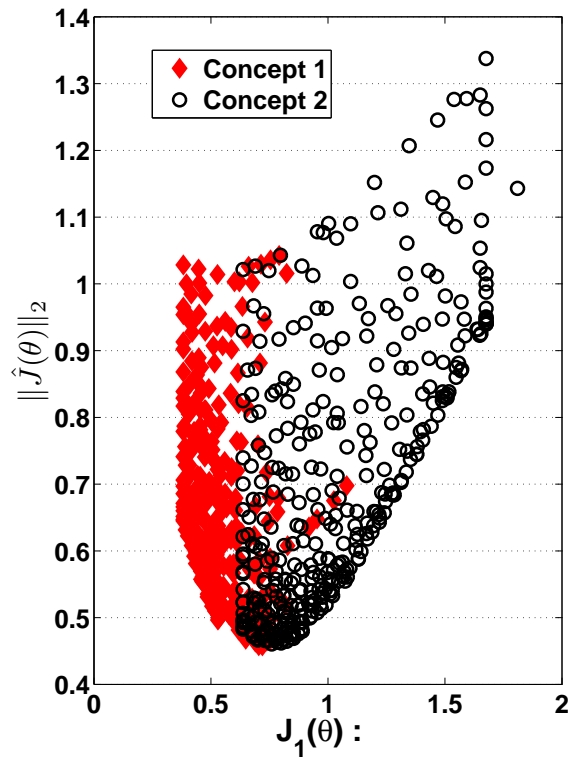

Mass of the brake [Kg.]

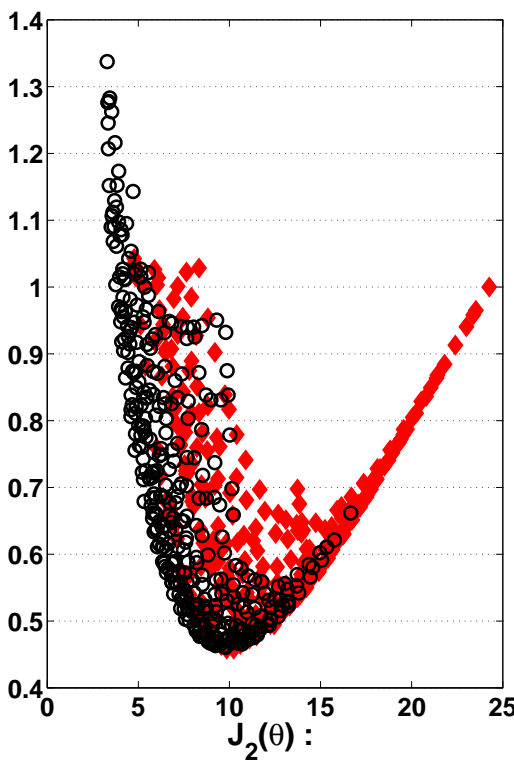

Stopping Time [secs.]

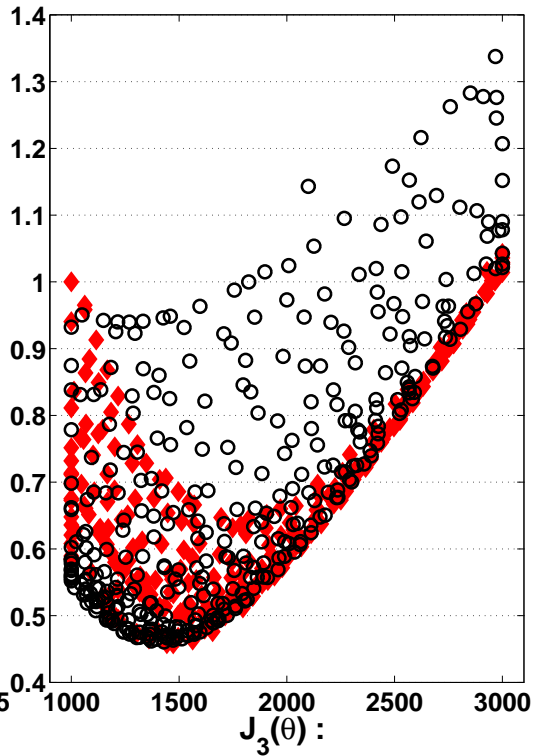

Engaging Force [N]

(b)

Figure 8: Concepts comparison using $\mathrm{LD} /\left\{J_{p 1}^{*}, J_{p 2}^{*}\right\} / Q\left(\boldsymbol{J}^{i}\left(\boldsymbol{\theta}^{i}\right), J_{p_{j}}^{*}\right)$ (a) and representation

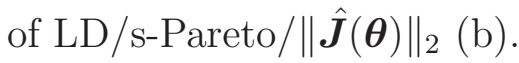


Notice how the analysis becomes more difficult as the objective space increases. In this example, using the $\mathrm{LD} /\left\{J_{p 1}^{*}, J_{p 2}^{*}\right\} / Q\left(\boldsymbol{J}^{i}\left(\boldsymbol{\theta}^{i}\right), J_{p_{j}}^{*}\right)$ visualization, it is possible to decide under which circumstances one concept will be preferred over another. For example, when the DM has a strong preference for one concept (due to its flexibility or because it is less complex), he can accept sub-optimal solutions. The following example will be used to show the flexibility of the $\mathrm{LD} /\left\{J_{p 1}^{*}, J_{p 2}^{*}\right\} / Q\left(\boldsymbol{J}^{i}\left(\boldsymbol{\theta}^{i}\right), J_{p_{j}}^{*}\right)$ visualization to built a Pareto front for a set of concepts filtered with preferences.

\subsection{Parametric controller design}

The next example is a parametric controller design $G(s)$ for the control benchmark proposed at the American Control Conference (ACC) [48]. The MOP statement described in [2] is used. It has six objectives: robust stability $\left(J_{1}(\boldsymbol{\theta})\right)$; maximum control effort $\left(J_{2}(\boldsymbol{\theta})\right)$, worst case settling time $\left(J_{3}(\boldsymbol{\theta})\right)$; noise sensitivity $\left(J_{4}(\boldsymbol{\theta})\right)$; nominal control effort $\left(J_{5}(\boldsymbol{\theta})\right)$; and nominal settling time $\left(J_{6}(\boldsymbol{\theta})\right)$. Two different controller structures $G_{1}(s)$ (design concept 1) and $G_{2}(s)$ (design concept 2 ) will be evaluated:

$$
\begin{aligned}
G_{1}(s) & =\frac{\theta_{1} s^{2}+\theta_{2} s+\theta_{3}}{s^{3}+\theta_{4} s^{2}+\theta_{5} s+\theta_{6}} \\
G_{2}(s) & =\frac{\theta_{1} s+\theta_{2}}{s^{2}+\theta_{3} s+\theta_{4}}
\end{aligned}
$$

subject to:

$$
\begin{array}{r}
-10 \leq \theta_{i} \leq 25 \\
J_{3}(\boldsymbol{\theta}) \leq 100 \\
J_{4}(\boldsymbol{\theta}) \leq 1 \\
J_{6}(\boldsymbol{\theta}) \leq 50
\end{array}
$$

The aims with this example are to:

- Demonstrate the viability of the approach with more than three objectives.

- Perform a simple interactive solution selection procedure.

In Figure 9, the $\mathrm{LD} /\left\{J_{p 1}^{*}, J_{p 2}^{*}\right\} / Q\left(\boldsymbol{J}^{i}\left(\boldsymbol{\theta}^{i}\right), J_{p_{j}}^{*}\right)$ visualization is shown. This figure shows that concept 2 better covers the objective space. Also, there are several solutions from concept 1 dominated by concept 2 .

An inspection of $\mathrm{LD} /\left\{J_{p 1}^{*}, J_{p 2}^{*}\right\} / Q\left(\boldsymbol{J}^{i}\left(\boldsymbol{\theta}^{i}\right), J_{p_{j}}^{*}\right)$ enables us to determine under which circumstances one concept will be preferred over another. To show this flexibility, let's assume the DM has a strong preference for concept 2, due to its simplicity and implementation 
facilities. Although concept 1 has a larger coverage, the DM observes that such covering implies a strong degradation in objective $J_{4}(\boldsymbol{\theta})$. Also, given the preference for implementing concept 2 , the DM decides that small improvements of concept 1 over concept 2 are not justifiable. With this, the DM can build a Pareto front for a set of concepts filtered with his/her preferences (fs-Pareto front). For this particular example, lets say:

- Concept 2 is preferred over concept 1, when covering the same objective space (i.e., if both concepts have the same type of objective tradeoff, then concept 2 will be always be selected).

- Solutions from concept 2 will be removed from the fs-Pareto front only if $Q\left(J^{1}\left(\boldsymbol{\theta}^{\mathbf{1}}\right), J_{p 2}^{*}\right)>$ 1.10 (i.e., there is an acceptance region of $10 \%$ for a solution from concept 2 even if it is dominated by $\left.\boldsymbol{J}_{p 1}^{*}\right)$. That is, we would accept some sub-optimal solutions given the simplicity of the design concept.

- Solutions from concept 2 will be inserted in the fs-Pareto front only if $Q\left(J^{2}\left(\boldsymbol{\theta}^{\mathbf{2}}\right), J_{p 1}^{*}\right)<$ $\frac{1}{1.10}$ (i.e. a minimum improvement over concept 2 is required for any solution from concept 1 to be considered.)

- The DM would like to incorporate the preferences defined in Table 3 to facilitate its selection procedure.

Table 3: Preference matrix for Example 3.

\begin{tabular}{crrrr}
\hline & \multicolumn{1}{c}{$\leftarrow$ HD $\rightarrow$ Highly } \\
Desirable & Desirable & Tolerable \\
Objective & $J_{i}^{0}$ & $J_{i}^{1}$ & $J_{i}^{2}$ & $J_{i}^{3}$ \\
\hline Robust performance & -1.00 & -0.04 & -0.02 & -0.001 \\
Maximum control effort & 0.00 & 0.30 & 0.40 & 0.500 \\
Worst case settling time & 0.00 & 40.00 & 60.00 & 80.000 \\
Noise sensitivity & 0.00 & 0.50 & 0.60 & 0.700 \\
Nominal control effort & 0.00 & 0.30 & 0.40 & 0.500 \\
Nominal settling time & 0.00 & 30.00 & 40.00 & 50.000 \\
\hline
\end{tabular}

With the aforementioned preferences, an fs-Pareto front is built. This front merges solutions from both concepts with a given set of preferences of the DM. In Figure 10 the $\mathrm{LD} /$ fs-Pareto $/\|\hat{\boldsymbol{J}}(\boldsymbol{\theta})\|_{2}$ visualization is shown. From this point onwards, the usual analysis on LD/front/measure using the LD-Tool [2] can be performed. For example, a solution (designed with a star in the aforementioned figure) could be selected to appreciate tradeoff and performance when compared with concept 1 . 

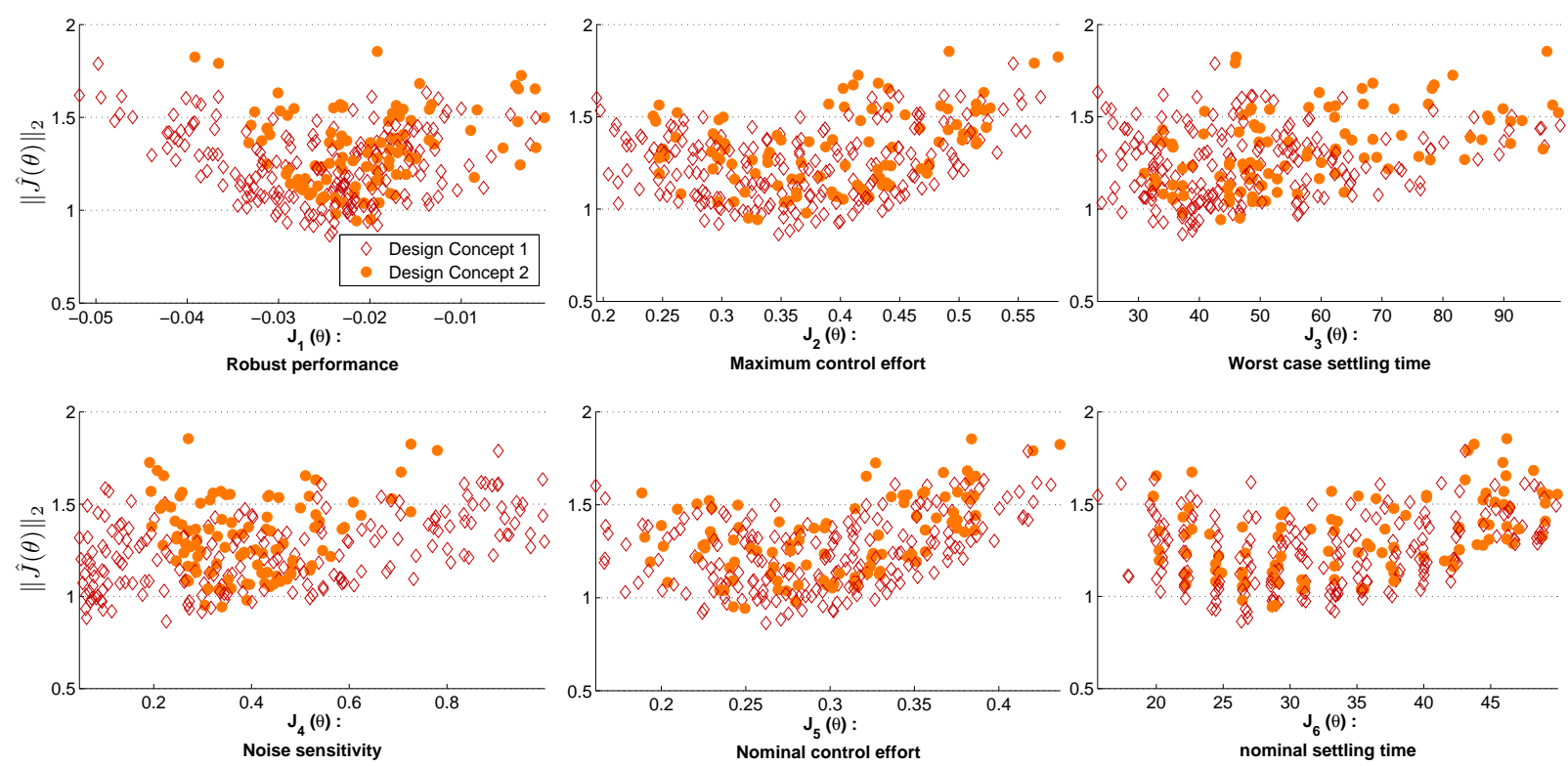

(a) $\mathrm{LD} /\left\{J_{p 1}^{*}, J_{p 2}^{*}\right\} /\|\hat{\boldsymbol{J}}(\boldsymbol{\theta})\|_{2}$
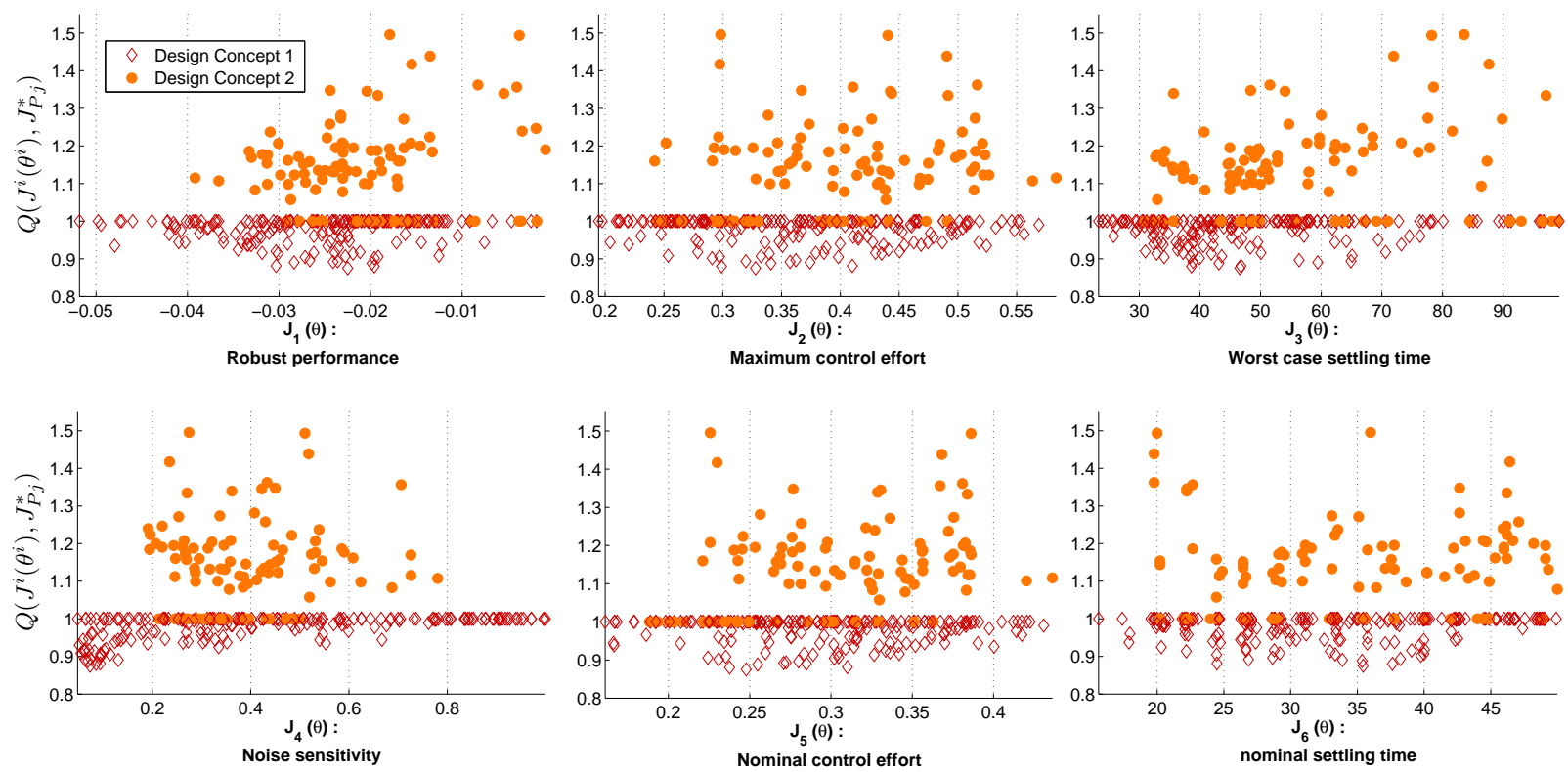

(b) $\mathrm{LD} /\left\{J_{p 1}^{*}, J_{p 2}^{*}\right\} / Q\left(\boldsymbol{J}^{i}\left(\boldsymbol{\theta}^{i}\right), J_{p_{j}}^{*}\right)$

Figure 9: Objective exchange visualization for example 3. 
It is important to notice that, even with a large dimensional objective space, the LD visualization remains useful for design alternative selection and concept comparison. Such a filter over the s-Pareto front facilities the DM analysis task, by allowing her/him to analyze the region and characteristics that are important according to her/his preferences.

\subsection{MOEA performance evaluation}

The final example is a proposal for MOEA visual comparison using the proposed approach. This example is used to:

- Make a proposal for MOEA visual comparison with more than three objectives.

- Show how the comparison of three or more design concepts could be performed.

Three different design concepts (MOEAs) are used to calculate a Pareto front approximation for problem WFG6 (with 32 decision variables and five objectives) from the benchmark test suit for multi-objective optimization described in [18]. Design concepts to be used are:

Concept 1: A DE algorithm with the diversity/pertinency mechanism described in [46].

Concept 2: A standard GA for multi-objective optimization.

Concept 3: A standard DE algorithm for multi-objective optimization.

In all cases, a target vector solution is defined at $\boldsymbol{J}(\boldsymbol{\theta})=[1.30,2.24,2.79,5.24,8.24]$. The GA algorithm uses a basic penalty-function mechanism for this purpose. A total of 25 independent runs for each design concept was evaluated and 2000 function evaluations are used.

In order to keep simplicity as a requirement, all the proposals will be compared with a Pareto front for reference $J_{p}^{r e f}$ (design concept 0) to avoid the comparison by pairwise design concepts. In this case, it is straightforward to generate such a Pareto front, since it is known. In other instances, a Pareto front for reference could be generated by merging all the solutions obtained and using a dominance filter. This would be helpful to visualize the degree of estrangement of design alternatives in a given design concept from the reference front.

To evaluate the average performance of the design concepts, the $m$-dimensional attainment surface is calculated as indicated in [21] 9 . The attainment surface at $50 \%\left(\widetilde{J_{p i}^{*}}{ }^{50 \%}\right)$ is calculated for the design concept comparison. In Figure 11 the $\operatorname{LD} /\left\{{\widetilde{J_{p 1}^{*}}}^{50 \%},{\widetilde{J_{p 2}^{*}}}^{50 \%}, \widetilde{J}_{p 3}^{*} 50 \%\right\}$ / $Q\left(\boldsymbol{J}^{i}\left(\boldsymbol{\theta}^{i}\right), J_{p 0}^{*}\right)$ visualization is shown. It is possible to appreciate that design concepts 2 and 3 (circles and points respectively) show almost the same performance in approximating the Pareto front. Concept 1 (squares) shows a better performance, since it is closer to 1 (the Pareto front for reference); furthermore, several solutions seem to be in the Pareto front, or

\footnotetext{
${ }^{9} \mathrm{C}$ code freely available at http://dbkgroup.org/knowles/plot_attainments/
} 

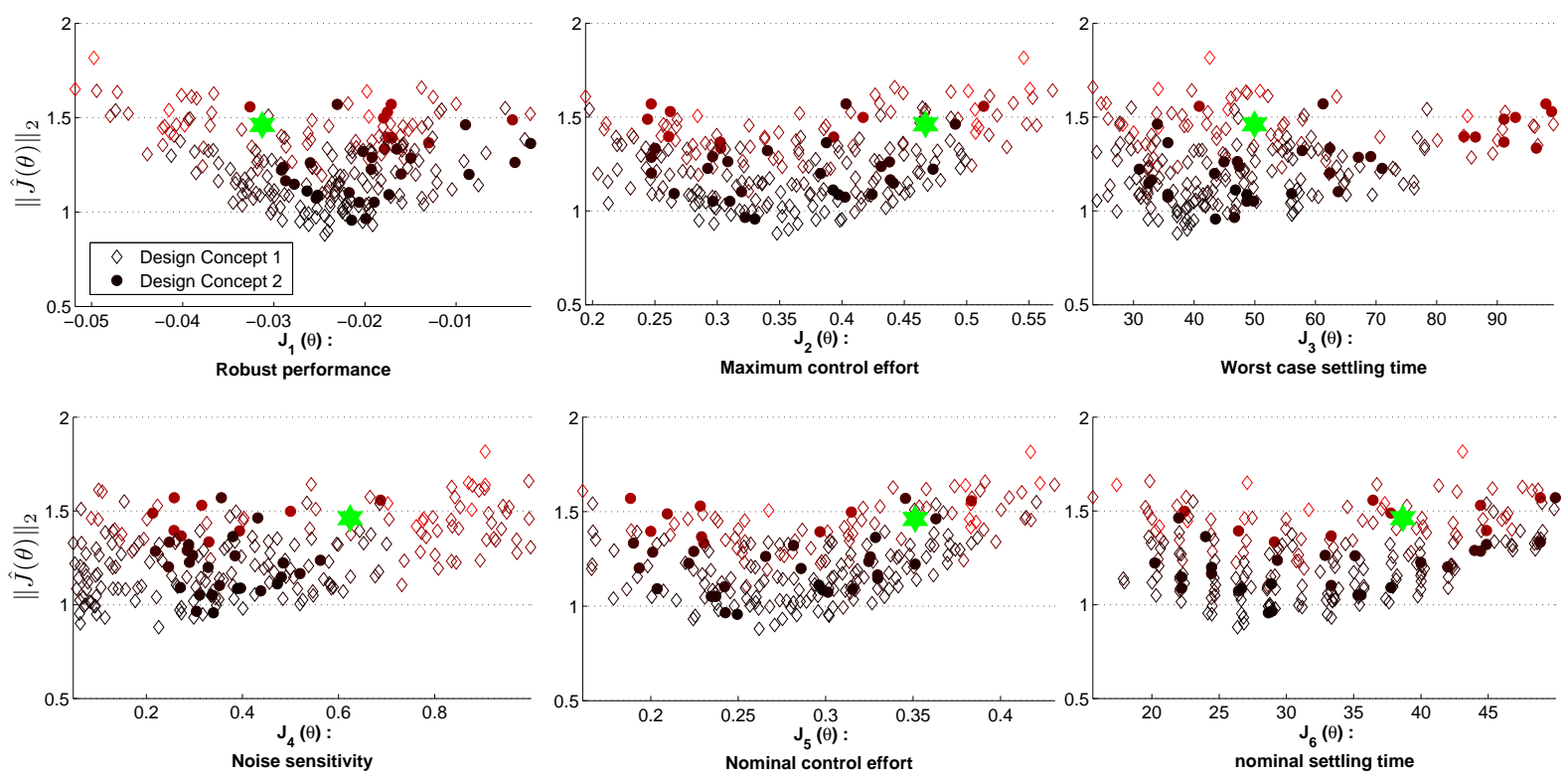

(a) LD/fs-Pareto/ $\|\hat{\boldsymbol{J}}(\boldsymbol{\theta})\|_{2}$
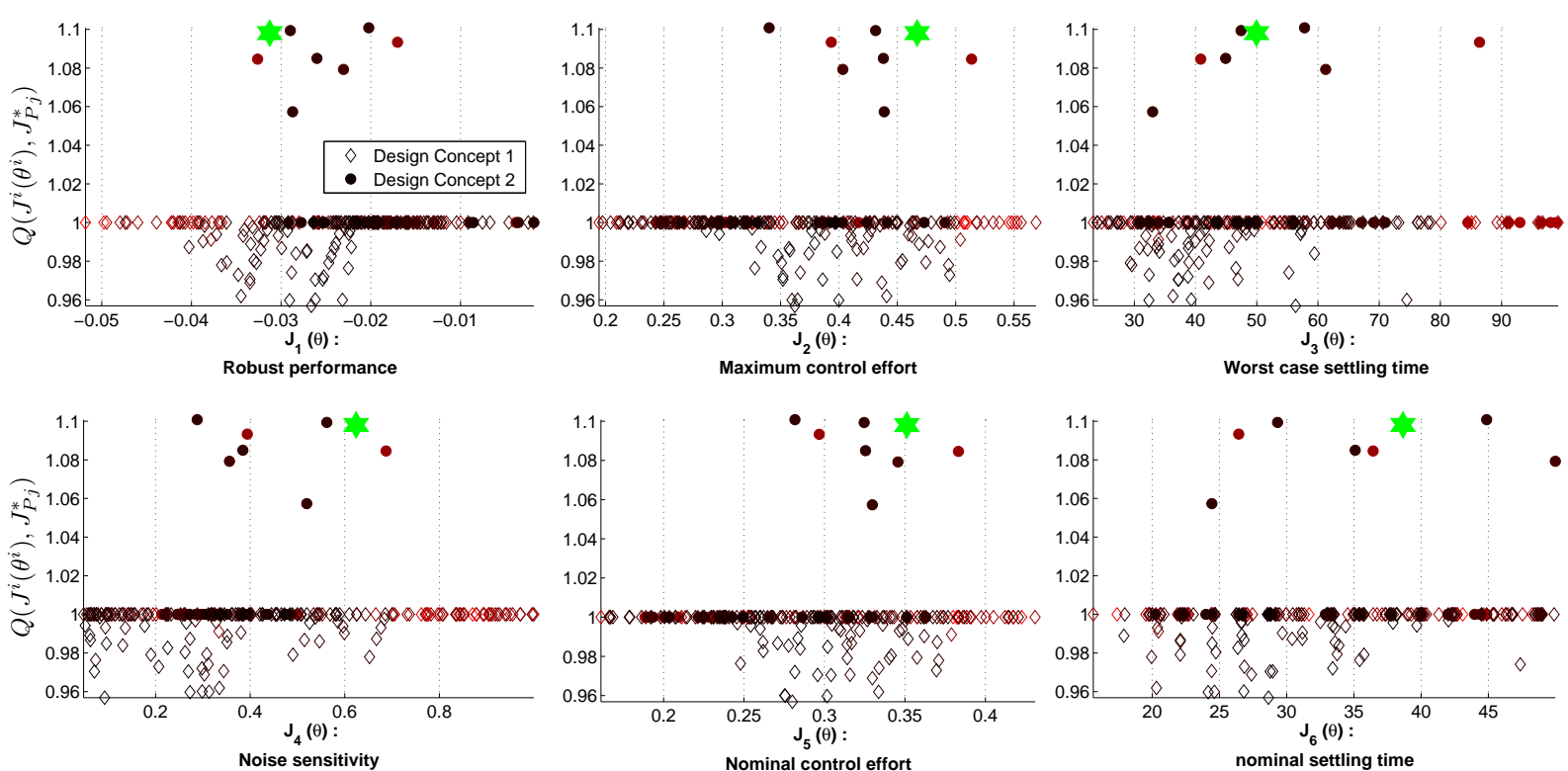

(b) LD/fs-Pareto $/ Q\left(\boldsymbol{J}^{i}\left(\boldsymbol{\theta}^{i}\right), J_{p_{j}}^{*}\right)$

Figure 10: Visualization with preferences (see table 3) for example 3. The darker the solution, the most preferable according to the DM's preferences. 
at least, are not dominated by the Pareto front for reference $J_{p 0}^{*} 10$. This graphical inspection, as noticed in [21] is helpful because it is important to have a visual validation for MOEA performance evaluation when other metrics are used (such as hypervolume for instance). Notice that whilst the performance metrics by themselves give an overall-quantitative measure on Pareto front performance, this graphical inspection enables a visualization and comparison of the algorithm performance by sectors.

\section{Conclusions}

In this work, a new quality measurement $Q\left(J^{i}\left(\boldsymbol{\theta}^{i}\right), J_{p j}^{*}\right)$ was defined to perform concept comparison using level diagrams (LD). With this measure, and the flexibility of LD to visualize and analyze $m$-dimensional Pareto fronts, the DM is able to obtain a better insight into objective tradeoffs for different concepts. This was validated in a set of multi-criteria decision making problems of 2,3,5 and 6 objectives.

Furthermore, with the $Q\left(J^{i}\left(\boldsymbol{\theta}^{i}\right), J_{p j}^{*}\right)$ quality measurement it is possible to have a measure of alternative design improvements between design concepts. With this feature, it is possible to build a Pareto front for a set of concepts filtered (fs-Pareto) with the DM preferences. In this fs-Pareto front, the DM can accept sub-optimal solutions for one concept when for example, the improvement of one concept over another is small, and the use of the s-Pareto optimal solution is not justifiable.

Finally, the $Q\left(J^{i}\left(\boldsymbol{\theta}^{i}\right), J_{p j}^{*}\right)$ quality measurement is used to test multi-objective optimization algorithms since it can measure the improvement of one algorithm over another. Although such evaluation can be done by means of the original $I_{\epsilon}$ binary indicator that calculates a single scalar for each Pareto front; with the $Q\left(J^{i}\left(\boldsymbol{\theta}^{\boldsymbol{i}}\right), J_{p j}^{*}\right)$ quality measurement it is possible to analyze the regions in the objective space where an algorithm performs better, thereby obtaining a better understanding of its weaknesses and strengths. This characteristic could be desirable when the algorithms are evaluated with real-world application problems.

\section{Appendix A. Supplementary Video}

Examples on usability and interactivity of LD visualization by means of the LD-tool are provided at: http://cpoh.upv.es/es/investigacion/software/item/52-ld-tool.html.

\section{Appendix B. sp-MODE}

The spMODE algorithm [35, 39] is a MOEA used to calculate a Pareto front approximation $J_{P}^{*}$. It is based on the differential evolution [45, 44, 9] technique and uses an external file to store the best solutions found and the extreme values for each objective. To handle the inclusion/exclusion of a solution in such repository, it uses a spherical pruning mechanism in the objective space to reduce the size of $J_{P}^{*}$. A general pseudo-code using spherical pruning is presented in Algorithm I.

\footnotetext{
${ }^{10} J_{p 0}^{*}$ is calculated with 1000 solutions uniformly distributed in the known Pareto set.
} 

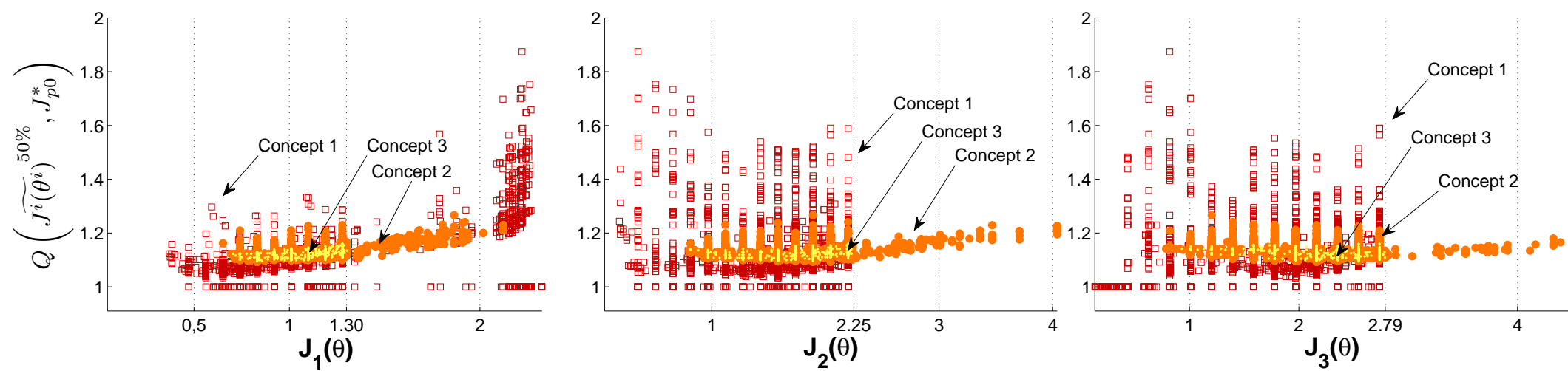

N
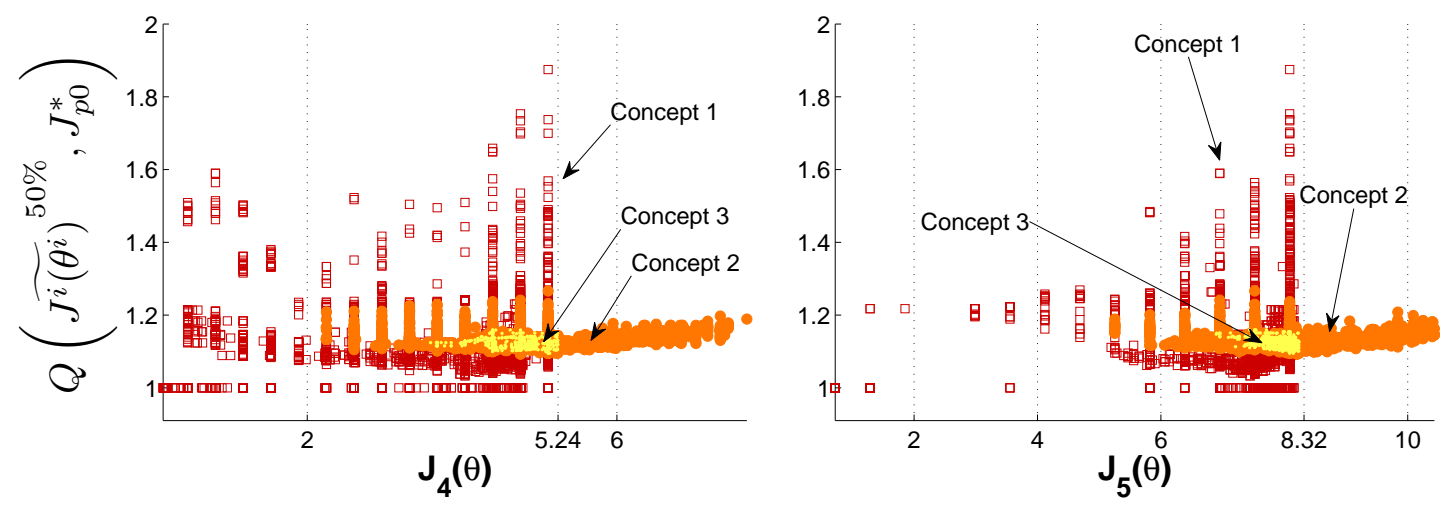

Figure 11: Visual comparison among MOEAs using LD/ $\left\{{\widetilde{J_{p 1}^{*}}}^{50 \%},{\widetilde{J_{p 2}^{*}}}^{50 \%},{\widetilde{J_{p 3}^{*}}}^{50 \%}\right\} / Q\left(\boldsymbol{J}^{i}\left(\boldsymbol{\theta}^{i}\right), J_{p 0}^{*}\right)$. 


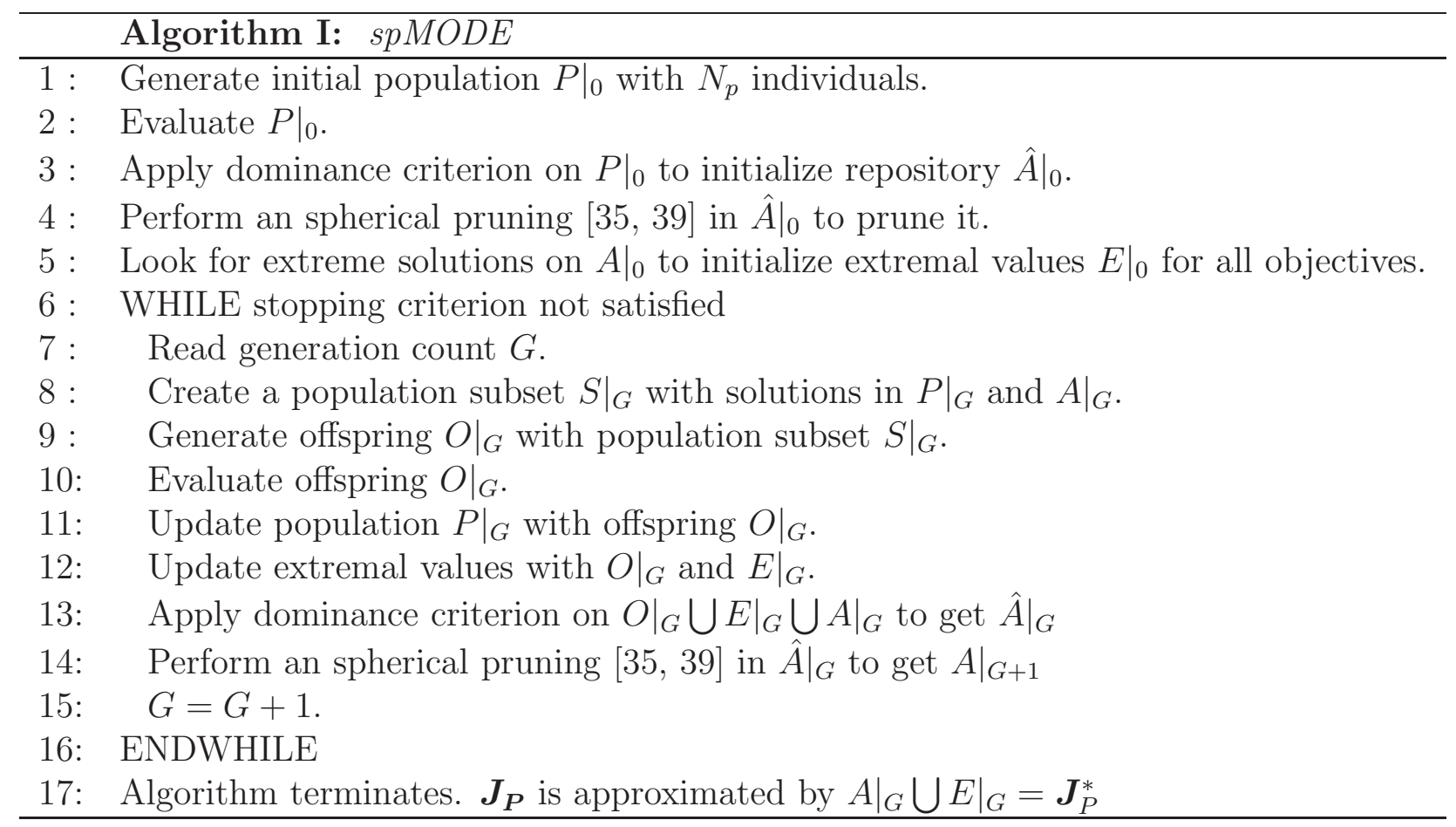

\section{Acknowledgment}

This work was partially supported by the FPI-2010/19 grant and project PAID-06-11 from the Universitat Politècnica de València and by projects ENE2011-25900, TIN201128082 (Spanish Ministry of Science and Innovation) and GV/2012/073 (Generalitat Valenciana).

[1] M. Behzadian, S.K. Otaghsara, M. Yazdani, J. Ignatius, A state-of the-art survey of TOPSIS applications, Expert Systems with Applications (2012) -.

[2] X. Blasco, J. Herrero, J. Sanchis, M. Martínez, A new graphical visualization of ndimensional Pareto front for decision-making in multiobjective optimization, Information Sciences 178 (2008) 3908 - 3924.

[3] P. Bonissone, R. Subbu, J. Lizzi, Multicriteria decision making (mcdm): a framework for research and applications, Computational Intelligence Magazine, IEEE 4 (2009) 48 -61 .

[4] R. Cela, M. Bollaín, New cluster mapping tools for the graphical assessment of nondominated solutions in multi-objective optimization, Chemometrics and Intelligent Laboratory Systems 114 (2012) $72-86$.

[5] Y. Chen, X. Zou, W. Xie, Convergence of multi-objective evolutionary algorithms to a uniformly distributed representation of the Pareto front, Information Sciences 181 (2011) $3336-3355$. 
[6] C.A.C. Coello, G.B. Lamont, Applications of Multi-Objective evolutionary algorithms, World scientific publishing, advances in natural computation vol. 1 edition, 2004.

[7] C.A.C. Coello, D.V. Veldhuizen, G. Lamont, Evolutionary algorithms for solving multiobjective problems, Kluwer Academic press, 2002.

[8] I. Das, J. Dennis, Normal-boundary intersection: a new method for generating the Pareto surface in non-linear multicriteria optimization problems, SIAM J. Optim. 8 (1998) $631-657$.

[9] S. Das, P.N. Suganthan, Differential evolution: A survey of the state-of-the-art, Evolutionary Computation, IEEE Transactions on PP (2010) 1 -28.

[10] K. Deb, A. Pratap, S. Agarwal, T. Meyarivan, A fast and elitist multiobjective genetic algorithm: NSGA-II, IEEE Transactions on Evolutionary Computation 6 (2002) 124 141.

[11] E. Fernandez, E. Lopez, F. Lopez, C.A.C. Coello, Increasing selective pressure towards the best compromise in evolutionary multiobjective optimization: The extended NOSGA method, Information Sciences 181 (2011) 44 - 56.

[12] J. Figueira, S. Greco, M. Ehrgott, Multiple criteria decision analysis: State of the art surveys, Springer international series, 2005.

[13] C. Fonseca, P. Fleming, Genetic algorithms for multiobjective optimization: formulation, discussion an generalization., Proceedings of the fifth international conference on genetic algorithms. (1993) $416-423$.

[14] W. Gong, Z. Cai, L. Zhu, An efficient multiobjective Differential Evolution algorithm for engineering design, Structural and Multidisciplinary Optimization 38 (2009) 137 157. 10.1007/s00158-008-0269-9.

[15] A. Hajiloo, N. Nariman-zadeh, A. Moeini, Pareto optimal robust design of fractionalorder PID controllers for systems with probabilistic uncertainties, Mechatronics (2012) - .

[16] A.G. Hernández-Díaz, L.V. Santana-Quintero, C.A.C. Coello, J. Molina, Paretoadaptive $\epsilon$-dominance, Evolutionary Computation (2007) 493 - 517.

[17] J. Herrero, M. Martínez, J. Sanchis, X. Blasco, Well-distributed Pareto front by using the $\epsilon$-MOGA evolutionary algorithm., in: F.S. et al. (Ed.), Computational and Ambient Intelligence, volume LNCS 4507, Springer-Verlag, 2007, pp. $292-299$.

[18] S. Huband, P. Hinstong, L. Barone, L. While, A review of multiobjective test problems and a scalable test problem toolkit, IEEE Transactions on evolutionary computation 10 (2006) $477-506$. 
[19] A. Inselberg, The plane with parallel coordinates, The Visual Computer 1 (1985) 69-91. 10.1007/BF01898350.

[20] A. Inselberg, Parallel Coordinates: Visual Multidimensional Geometry and Its Applications, Springer, 2009.

[21] J. Knowles, A summary-attainment-surface plotting method for visualizing the performance of stochastic multiobjective optimizers, in: Intelligent Systems Design and Applications, 2005. ISDA '05. Proceedings. 5th International Conference on, pp. 552 557.

[22] J. Knowles, L. Thiele, E. Zitzler., A tutorial on the performance assessment of stochastic multiobjective optimizers, Technical Report TIK report No. 214, Computer Engineering and networks laboratory. ETH Zurich, Feb. 2006.

[23] J.B. Kollat, P. Reed, A framework for visually interactive decision-making and design using evolutionary multi-objective optimization (VIDEO), Environmental Modelling \& Software 22 (2007) 1691 - 1704.

[24] K. Li, S. Kwong, J. Cao, M. Li, J. Zheng, R. Shen, Achieving balance between proximity and diversity in multi-objective evolutionary algorithm, Information Sciences 182 (2012) 220 - 242. Nature-Inspired Collective Intelligence in Theory and Practice.

[25] A. Lotov, K. Miettinen, Visualizing the Pareto frontier, in: J. Branke, K. Deb, K. Miettinen, R. Slowinski (Eds.), Multiobjective Optimization, volume 5252 of Lecture Notes in Computer Science, Springer Berlin / Heidelberg, 2008, pp. 213-243. 10.1007/978-3540-88908-3 9 .

[26] M. Martínez, J. Herrero, J. Sanchis, X. Blasco, S. García-Nieto, Applied Pareto multiobjective optimization by stochastic solvers, Engineering applications of artificial intelligence 22 (2009) $455-465$.

[27] M.A. Martínez, S. García-Nieto, J. Sanchis, X. Blasco, Genetic algorithms optimization for normalized normal constraint method under Pareto construction, Advances in engineering software 40 (2009) $260-267$.

[28] C.A. Mattson, A. Messac, Pareto frontier based concept selection under uncertainty, with visualization, Optimization and Engineering 6 (2005) 85-115. 10.1023/B:OPTE.0000048538.35456.45.

[29] A. Messac, A. Ismail-Yahaya, C. Mattson, The normalized normal constraint method for generating the Pareto frontier, Structural and multidisciplinary optimization (2003) $86-98$.

[30] A. Messac, C. Mattson, Generating well-distributed sets of Pareto points for engineering design using Physical Programming, Optimization and Engineering 3 (2002) 431-450. 10.1023/A:1021179727569. 
[31] K.M. Miettinen, Nonlinear multiobjective optimization, Kluwer Academic Publishers, 1998.

[32] M. Munro, B. Aouni, Group decision makers' preferences modelling within the goal programming model: An overview and a typology, Journal of Multi-Criteria Decision Analysis (2012) In press.

[33] A. Osyczka, S. Kundu, A modified distance method for multicriteria optimization, using genetic algorithms, Computers \& Industrial Engineering 30 (1996) 871 - 882.

[34] B. Qu, P. Suganthan, Multi-objective evolutionary algorithms based on the summation of normalized objectives and diversified selection, Information Sciences 180 (2010) 3170 - 3181. Including Special Section on Virtual Agent and Organization Modeling: Theory and Applications.

[35] G. Reynoso-Meza, Design, coding and implementation of a multiobjective optimization algorithm based on Differential Evolution with spherical pruning: applications for system identification and controller tuning., Master's thesis, Universitat Politècnica de València., 2009.

[36] G. Reynoso-Meza, X. Blasco, J. Sanchis, Diseño multiobjetivo de controladores PID para el benchmark de control 2008-2009, Revista Iberoamericana de Automática e Informática Industrial 6 (2009) 93 - 103.

[37] G. Reynoso-Meza, S. García-Nieto, J. Sanchis, X. Blasco, Controller tuning using multiobjective optimization algorithms: a global tuning framework, IEEE Transactions on Control Systems Article in press. (2012).

[38] G. Reynoso-Meza, J. Sanchis, X. Blasco, H. Juan M., Multiobjective evolutionary algortihms for multivariable PI controller tuning, Expert Systems with Applications 39 (2012) $7895-7907$.

[39] G. Reynoso-Meza, J. Sanchis, X. Blasco, M. Martínez, Multiobjective design of continuous controllers using differential evolution and spherical pruning, in: C.D. Chio, S. Cagnoni, C. Cotta, M. Eber, A. Ekárt, A. I.Esparcia-Alcaráz, C.K. Goh, J. J.Merelo, F. Neri, M. Preuss, J. Togelius, G. N.Yannakakis (Eds.), Applications of Evolutionary Computation, Part I, volume LNCS 6024, Springer-Verlag, 2010, pp. 532 - 541.

[40] S. Ruzika, M. Wiecek, Successive approach to compute the bounded Pareto front of practical multiobjective optimization problems, SIAM J. Optim. 20 (2009) 915 - 934.

[41] H. Safikhani, M. Akhavan-Behabadi, N. Nariman-Zadeh, M.M. Abadi, Modeling and multi-objective optimization of square cyclones using cfd and neural networks, Chemical Engineering Research and Design 89 (2011) 301 - 309. 
[42] J. Sanchis, M. Martínez, X. Blasco, J.V. Salcedo, A new perspective on multiobjective optimization by enhanced normalized normal constraint method, Structural and multidisciplinary optimization (2008) $537-546$.

[43] J. Sanchis, M.A. Martínez, X. Blasco, G. Reynoso-Meza, Modelling preferences in multiobjective engineering design, Engineering Applications of Artificial Intelligence 23 (2010) $1255-1264$.

[44] R. Storn, Sci: Differential evolution research: Trends and open questions, volume LNCS 143, Springer, Heidelberg, 2008, pp. $1-31$.

[45] R. Storn, K. Price, Differential evolution: A simple and efficient heuristic for global optimization over continuous spaces, Journal of Global Optimization 11 (1997) 341 359.

[46] L. Thiele, K. Miettinen, P.J. Korhonrn, J. Molina, A preference-based evolutionary algorithms for multi-objective optimization, Evolutionary Computation (2009) 411 436.

[47] Y. Wang, Y. Yang, Particle swarm optimization with preference order ranking for multiobjective optimization, Information Sciences 179 (2009) 1944 - 1959. Special Section: Web Search.

[48] B. Wie, D.S. Bernstein, Benchmark problems for robust control design, Journal of Guidance, Control and Dynamics. (1992) 1057 - 1059.

[49] J. Zhu, K.W. Hipel, Multiple stages grey target decision making method with incomplete weight based on multi-granularity linguistic label, Information Sciences 212 (2012) 15 32 .

[50] E. Zio, R. Bazzo, Level diagrams analysis of pareto front for multiobjective system redundancy allocation, Reliability Engineering \& System Safety 96 (2011) 569 - 580.

[51] E. Zio, R. Bazzo, Multiobjective reliability allocation in multi-state systems: Decision making by visualization and analysis of pareto fronts and sets, in: A. Lisnianski, I. Frenkel (Eds.), Recent Advances in System Reliability, Springer Series in Reliability Engineering, Springer London, 2012, pp. 195-208.

[52] E. Zio, R. Razzo, Multiobjective optimization of the inspection intervals of a nuclear safety system: A clustering-based framework for reducing the pareto front, Annals of Nuclear Energy (2010) 798-812.

[53] E. Zitzler, L. Thiele, M. Laumanns, C. Fonseca, V. da Fonseca, Performance assessment of multiobjective optimizers: an analysis and review, Evolutionary Computation, IEEE Transactions on 7 (2003) $117-132$. 